Elsevier Editorial System(tm) for Composites Part A

Manuscript Draft

Manuscript Number:

Title: Microtomography on Polypropylene Foam under Dynamic Loading.

3D Analysis of Bead Morphology Evolution

Article Type: Research Paper

Section/Category:

Keywords:

Corresponding Author: Dr philippe viot, Ph. D.

Corresponding Author's Institution: ENSAM

First Author: philippe viot, $\mathrm{Ph} \mathrm{D}$

Order of Authors: philippe viot, Ph D; Erwan Plougonven; Dominique Bernard, Ph D

Manuscript Region of Origin:

Abstract: A dynamic crash loading experiment is performed on a polypropylene foam used in composite sandwich structures for safety applications. Several interrupted shocks are conducted, in between which microtomographic acquisitions are made, showing the evolution of the sample during its compression. This data can help construct and validate predictive models, although, because this material is multiscale (consitutive beads at the mesoscopic scale are made of microscopic closed cells), image processing is required to extract useful quantitative measurements. Such processing is described here, so as to determine a representative volume for each bead of the sample, in order to associate to each bead and to each stage of the compression values such as bead density. This can help build a predictive model at the mesoscopic scale. 


\title{
Microtomography on Polypropylene Foam under Dynamic Loading. 3D Analysis of Bead Morphology Evolution
}

\author{
Philippe Viot*, Erwan Plougonven**, Dominique Bernard** \\ * LAMEFIP, ENSAM de Bordeaux, Esplanade des arts et métiers, 33405 Talence Cedex, \\ France \\ ** Institut de Chimie de la Matière Condensée de Bordeaux ICMCB, CNRS/Université \\ Bordeaux I, 87, Av. Dr A.Schweitzer, 33608 PESSAC Cedex, France.
}

\begin{abstract}
A dynamic crash loading experiment is performed on a polypropylene foam used in composite sandwich structures for safety applications. Several in terrupted shocks are conducted, in between which microtomo graphic acquisitions are made, showing the evolution of the sample during its compression. This data can help construct and validate predictive models, although, because this material is multiscale (consitutive beads at the mesoscopic scale are made of microscopic closed cells), image processing is required to extract useful quantitative measurements. Such processing is described here, so as to determine a representative volume for each bead of the sample, in order to associate to each bead and to each stage of the compression values such as bead density. This can help build a predictive model at the mesoscopic scale.
\end{abstract}

KEYWORDS: polypropylene foam, dynamic compression, micro tomography, 3D image analysis

\section{Introduction}

Composite multi-layers or sandwiches are becoming widely used in many industrial sectors for producing structural parts. Compiling an exhau stive list of all types of applications is difficult, but if we consider the transport sector, we find composite sandwiches for the aeronautic industry in key parts (wing leading edge, rudders). In the railroad industry, these structures are often used for wagon decor panels. As for the automotive and motorcycle industries, they are used in passive safety gear (bumpers or helmets). In all these applications, the intrin sic properties of light weight and rigidity are used. These sandwiches, composed of a core of cellular material and two composite skins, are light (since their constituents are of low density), rigid in traction and compression (the composite materials have good mechanical properties) but also in bending since the foam core thickens the structure (and thus increases its quadratic moment while limiting its weight) and supports high bending moments. These properties are particularly interesting for producing functional structures that must sustain high stresses under normal conditions. During severe or extreme loadings (crashes or accidents), these structures must deform plasticly and absorbs the impact energy in order to protect either the rest of the structure or the passengers. In the case of a plane wing that can collide with a bird, the leading edge should be able to absorb the energy of the impact and lessen damage to the other structural elements. In a train accident, the composite decor panels must deform themselves if a passengers is thrown onto the structure. Finally, a car is the first passive safety element that protects the vehicle pas sen ger or pedestrian involved in the accident. It is thus imperative to control the respon se of composite sandwiches to high stran rates. Characterisation of the behaviour of the composite material and core cellular material 
under dynamic loadings is essential before considering numerical modelling of the real structure. The study we are particularly concerned with is the description of the cellular material behaviour during dynamic loading.

Many studies have been carried out at the macroscopic scale to characterise the behaviour of this type of material under dynamic stresses [1-5]. Empirical laws were proposed to describe more or less accurately the relation between stress and strain according to different parameters such as density and strain rate (eventually the temperature). The behaviour of cellular material generally includes three steps in compression: an elastic response, a plastic regime with an important deformation of the material and quasi-constant stress, and finally a densification. It is the stress plateau of the plastic phase that is of interest for passive security applications since the material can absorb a significant fraction of the impact kinetic energy. The macroscopic laws used in finite element codes can coarsely describe the macroscopic behaviour of cellular materials (in the elastic and stress plateau phases) but do not take in to account the localisations of the observed strains -and thus their heterogeneity. These laws, used in industry and numerically adjusted on the global stress curves, can be used to represent the behaviour of a large variety of cellular materials such as polymeric or aluminium foams. It is well known that for the former, the predominant phenomenon in its damage is the wall buckling of the constitutive cells whereas wall fractures can be observed in the latter. It is therefore essential to propose macroscopic behaviour models which take into account the physical phenomena observed at all scales (macroscopic scale, bead scale in the case of expanded polymeric foams and cell scale) of the cellular material structure.

This study is part of a work whose aim is to propose a behaviour model based on the physical phenomena observed on expanded polymeric foam during dynamic loading. The structure of the considered polypropylene foam is multi-scale: at the mesoscopic scale, as seen in figure $1 \mathrm{a}$, the material consists of porous polypropylene beads agglomerated during manufacturing, beads that are composed of thousands of small closed cells, shown in figure $1 \mathrm{~b}$ (microscopic scale). Evolution of this type of material under dynamic stress is thus foreseen by a multiscale description. It is obviously necessary to identify the global response of materials at the macroscopic scale for various strain rates, but also to investigate the response of the foam structure at the mesoscopic (that of the bead) and microscopic scales (that of the basic cell). Observation of the deformation of the meso- and microscopic walls (bead and cell) will be one of the elements that will help to establish phenomenological laws at these scales.

Homogenisation methods will then be used to build a model describing the foam response at the macroscopic scale. The first step of this methodology, described in this article, is the observation, the comprehension and the characterisation of the physical phenomena. This step is essential to reach the final objective of foam structure modelling. The following steps will be to represent the polymeric foam by a polyhedral structure tak ing into account the two meso- and microscopic scales of the material (figure 1c) and to derive a macroscopic model fitting experimental results with numerical simulation ones.

\section{Material and methodology}

\section{Polypropylene foam}

A polypropylene foam is investigated in this paper. The plates of cellular material are obtained by moulding; the expanded plastic foam beads are injected into a plate mould (700 $\mathrm{mm} \times 400 \mathrm{~mm} \times 24 \mathrm{~mm}$ ), where individual beads are fused together under steam heat and pressure. The average density of each plate intrinsically depends on the quantity of expanded beads injected into the mould. For this study, the density of the plate is $80 \mathrm{~kg} / \mathrm{m}^{3}$. The moulding process, particularly injector and vent positions, induces density heterogeneities. Horizontally, measurements on a large number of samples cut out from this plate show values as large as $92 \mathrm{~kg} / \mathrm{m}^{3}$. Vertically, i.e. through the thickness of the plate, density increases 
significantly near the surfaces $(12 \%)$, whereas in the central region it is relatively uniform. Consequently, the selected samples (10 $\mathrm{mm}$ in diameter and height) were taken from the centre of the plate.

\section{Methodology}

The physical phenomena observed on polymeric foams during dynamic compression has already been studied under specific cond itions [6]. After impact, the buckling of both cell and bead walls have been observed by SEM. A strong heterogeneity of the residual deformation can be seen. However, the main difficulty of these observations lies in the sample cutting. Complementary measurements were taken during impact with the use of optical acquisition apparatus and image processing techniques (high-speed camera, optical fibre spotlights and Digital Image Correlation software). A strong heterogeneity of the strain field was shown, and strain localisations appear in layers perpendicular to the loading direction. The material damage progresses close to these zones during the stress plateau [6,7]. However these observations are only possible on the free faces of the sample, implying that this method cannot estimate the strain field inside the foam structure.

In light of these first results, micro tomography was considered for observing the deformation inside the foam structure. Tomography is a non-invasive process of generating images of cross-sections from a series of transmission data acquired by illuminating the object from different directions. In the case of X-ray tomography, for each direction of illumination, the process is similar to radiography: the acquired transmission data gives a map of the rays attenuation. Figure 2 illustrates this: the centre object, consisting of an ellipse and a circle, is illuminated from two directions by a parallel beam, the acquired images are called projections, which are used to reconstruct cross-sections of the object. In reality, many more projections are needed for an accurate reconstruction ( 900 in our case).

The identification of the foam deformation and damage propagation in $3 \mathrm{D}$ from microtomography measurements needs an original dynamic test methodology [8]. The adopted experimental approach consists in carrying out sev eral interrupted impacts on a given sample using a drop tower, and acquiring a microtomogram in between each impact. The sample (diameter and height of $10 \mathrm{~mm}$ ) is scanned a first time before the first impact. During each compression, the deformation amplitude is limited to fixed values: $1 \mathrm{~mm}$ for the first impact and $2 \mathrm{~mm}$ for the following ones. Figure 3 shows on the stress-strain curves the different states the sample was in when acquisitions were made. The sample is main tained compressed and replaced on the microtomography setup for another acquisition (points A, B, C...figure 3). A second microtomogram is recorded when the sample is completely unloaded (points A',B', C'...). These operations (impact and $2 \mathrm{X}$-ray scans) are repeated until densification of the foam. The cellular material strain can then be evaluated from the 3D reconstructions at each stage of the dynamic test.

Microtomographic images presented in this paper have been obtained on the BM05 beam line at the European Synchrotron Radiation Facility (ESRF) in Grenoble (France), with a beam energy of $16 \mathrm{keV}$. The acquired projections are 2028x2048 pixels radiographs, with a pixel corresponding to $4.91 \mu \mathrm{m}$.

The $3 \mathrm{D}$ reconstruction us es pre- and post-processing to reduce artifacts and noise, such as hot spots or ring artifacts.

From these measurements, a first analysis was done in 2D. The deformation of beads located in a vertical section of the foam was calculated for each step of the experiment [8] and bead density was estimated. This first study shows that there is not a strong correlation between the density of beads and their volume deformation, meaning that bead density is not the principal parameter influencing the deformation heterogeneity. However, the arrangement of beads, their shapes and the geometry of the meso-structure seem to have an influence of the strain 
field. These results encourage us to complete the analysis of this sample by examining the en tire 3D volume and all the beads it contains, for two main reasons. Firstly, in a 2D analysis, it is necess ary to consider bead displacement out of the vertical section as negligible. This assumption seems to be verified globally but can introduce some error in the estimation of the deformation. In $3 \mathrm{D}$, th is hypothesis is not necessary; the volume strain is only calculated from the change of bead volume and the 3D result is necessarily more accurate. Secondly, the deformation calculation on the whole of the sample can confirm the first results obtained -in 2D- on a few number of beads. In 3D, the deformation of a larger number of beads of different density can be compared.

The main difficulty of a $3 \mathrm{D}$ analysis is to extract the bead walls from the multi-scale structure: figure 4 shows that, it is difficult even interactively to accurately position the walls. This problem is minimised for other types of foams that have been more extensively studied, such as metallic foams. Typical digital image filters are not sufficient to extract these complex walls: no ise reduction and thresholding produces structures with unrealistic porous zones and traces of cells or bubbles with thick walls, as illustrated in figure 5, which cannot be used to quantify bead volume changes or bead strain, let even to obtain a FE mesh of the foam morphology.

To overcome this, a new approach for identifying the bead structure was developed. The method requires a perspicacious sequence of typical image processing operations to extract a more accurate representation of the bead structure that is useable for calculating bead strain.

This paper presents the image analysis that was implemented to identify distinct regions in the microtomograms for each bead that makes up the foam. The method was applied at several steps of the loading and the evolution of bead strain can be estimated and compared to bead density.

\section{Image Analysis Methodology}

The purpose of this work is to understand the behaviour of polypropylene foam at the mesoscopic scale (scale of the beads), for which extracting pertinent data from the microtomographic images is required. To perform measurements on the beads, a representative volume in each one is determined. The final objective of this work is to follow the deformation of these Representative Bead Volumes (RBV) and to estimate the influence of bead density. This section describes the way these volumes are extracted.

The method consists in two steps: firstly, a series of filters applied on the reconstructed volumes gives approximate positions for the bead centres, and secondly each centre serves as starting point for a deformable surface algorithm, in which a triangulated surface placed inside a bead expands until coming in contact with bead walls. These surfaces are used to delimit representative bead volumes in the microto mo grams.

\subsection{Extracting approximate bead centres}

Estimating bead centre positions requires a sequence of basic filtering operators to be applied on the reconstructed image. Figure 6 summarises the filtering sequence, and figure 7 shows various intermediary results. First, the image is sub-sampled: each 4-pixel wide cube in the image is replaced by one pixel, dividing the size of the numerical volume by 64 (step a). This was done because of hardware limitations, but in no way affects the correctness of the method. The next operation partially removes phase contrast (due to X-ray diffraction at the interfaces between phases of the sample, an enhanced contrast appears around portions of 
bead and cell walls [10]) by removing darker areas: if a pixel $p$ has an intensity $i$ lower than the intensity $i_{0}$, equivalent to the attenuation of the air, then they are set to $i_{0}$ (step b). The reason for this is that at some point small features such as noise and microscopic cells need to be smoothed out, and if a gaussian filter is applied on the image containing phase constrast, i.e. light zon es next to dark ones, a smoothing operator will level these areas to that of the rest of the image. A gaussian filter (step c) is then applied to remove noise and microscale features inside the beads. The next op erator (step d), a gradient (the Sobel norm was used), highlights the bead edges.

The next part consists in extracting most pixels from the bead walls, which is where the gradient is higher. Operations described here are based on notions of digital topology [10]. First a hysteresis thresholding is applied (i.e. the image is thresholded with a high and low value, giving two binarised images, respectively $\mathrm{I}_{\mathrm{h}}$ and $\mathrm{I}_{\mathrm{l}}$, then a geodesic dilation is applied to $\mathrm{I}_{\mathrm{h}}$ in $\mathrm{I}_{1}[11]$ ), which makes for a better binarisation than a classical thresholding since the bead walls are connected (step e). The thresholds are chosen so as to extract enough of the bead walls to obtain a single connected component for these pixels, and as little as possible of the inside of the beads. The result does not contain all the pixels of the bead walls and contains a few noticeable cells around and inside the beads. The cells around the bead walls hardly affect the bead cen tre approximation, but the ones inside the cells should be removed, which is done by extracting the largest connected component (step f). Afterwards, to avoid the unreconstructed area of the image to influence the ensuing operations, a cylin drical mask is applied (step g): the resulting image has foreground pixels belonging either to the unreconstructed areas of the image or the bead wall borders.

The resulting image at step $g$ is used to compute a distance map (i.e. the in tensity of each pixel of the background is set to its distance from the foreground), in which pixels close to the bead centre are darker, and those in and around the bead walls are lighter (step h). The distance map is then binarised by a thresholding operator (with a threshold value high enough to disconnect all the beads, since portions of the bead walls were missing from the image used for the distance map). In the binar ised image, smaller connected components that appear at junctions between beads due to artifacts from the previous operations are removed. Finally an image with as many connected components as there are beads in the sample is obtained. What is finally defined as bead centres is the barycentres of these connected components. Figure 8 depicts this result: an isosurface of the distance map is drawn, with the isosurface value corresponding to the threshold used.

\subsection{Deformable surfaces}

Each bead centre serves as initial position for a deformable surface: a closed triangulated surface, initially having a spherical shape, is placed inside the bead and expands under given conditions such that it converges towards pixels of higher intensity, i.e. bead walls.

It might be suggested that the volumes delimited by the isosurfaces used to define the centres can be directly u sed to estimate bead strain. However, the volumes of the isosurfaces identified are not large enough to be considered representative of the bead (the set of isosurfaces only en compass about $35 \%$ of the sample volume). By using a deformable surface algorithm, larger portions of the bead interiors are determined (around 75\%) which account for a better bead representativity.

To obtain an RBV, the implemented algorithm is based on the works of Delingette et al. $[12,13]$. The evolution of the surface, which is a 3-simple mesh (a mesh in which each vertex is contained in exactly 3 facets), is an explicit model, i.e. the evolution is calculated at each step $t$, and is made by subjecting each vertex $p$ of the mesh to a second order Newtonian law 
of motion $\sum_{m=d, i, v, r} \vec{F}_{m}=m \vec{a}$, where $\vec{F}_{m}$ represents several forces, classified as internal and external to the mesh, $m$ a mass assigned to the vertex $p$ and $\vec{a}$ its acceleration. The forces are defined as: a dilation force $\vec{F}_{d}=k_{d} \vec{N}$ expands the surface, an opposite intensity force $\vec{F}_{i}=-k_{i} \vec{N}$, which increases when the vertex is inside a high-intensity region of the image, contracts the surface, and a viscosity force $\vec{F}_{v}=k_{v} \vec{V}$ adds friction to the movement to avoid divergence. The constants $k_{x}$ are manually set to optimise the convergence of the surface, the vectors $\vec{V}$ and $\vec{N}$ are respectively the speed of $p$ and the normal of the plane defined by the three neighbours $n_{1}, n_{2}$ and $n_{3}$ of $p$ (the neighbours are oriented so that the normal always points outwards). The internal force is a regularisation force $\vec{F}_{r}=k_{r} \cdot(\vec{g}+\widetilde{d} \vec{N}-\vec{p})$ that smoothes the curvature of the surface. The curvature is based on the elevation $d$ of the vertex, which is controlled by its simplex angle $\varphi$, as shown in figure 9 . The value $\tilde{d}$ represents the elevation, above the cen tre of gravity of the triange $n_{1} n_{2} n_{3}$, of a point that has a simplex angle $\widetilde{\varphi}=\sin ^{-1}\left(r \sum_{j \in[1 ; 3]} \frac{\sin \varphi_{j}}{r_{j}}\right)$, where $r$ is the radius of the circle circumscribing the triange, and $\varphi_{j}$ and $r_{j}$ are respectively the simplex angle of $n_{j}$ and radius of the circle circumscribing the neighbours of $n_{j}$. Convergence is achieved when, for each vertex, all forces are balanced and speed drops to zero.

An essential factor for this approach is the choice of the image that defines the force field $\vec{F}_{i}$. This image should contain higher intensity values near the areas where the deformable mesh is to converge. Altough the initial image, the reconstructed microtomogram, appears to have suitable features for this process, the amount of no ise, significant cell wall intensity and higher intensity near the sample periphery make it an in appropriate choice. The best results were obtained with a smoothed gradient, as in step $d$ of figure 7 . To avoid surfaces expanding outside of the sample bounding volume, a cylindrical mask was applied to this image, as in step g. Results of this algorithm (obtained from the image of the sample before impact) is shown in figure 10: each bead is represented by a surface, and compared to figure 8 , these surfaces are more tightly packed. There is still however a notable intersticial volume in between the beads, which has no physical significance: in the material, foam beads are in contact with each other and no space exists between them (except in some regions neighbour to three or more beads). This is due both to areas where bead delimitations are not marked by strong intensity variations, and to cell walls which are as pronounced as bead walls (even to the naked eye it is sometimes unclear where bead walls are located, see figure 4).

Nevertheless, the present task is not to completely encompass the bead volumes, but to obtain an RBV.

\section{Results}

This methodology is applied on the polypropylene foam sample and for each stage of dynamic loading. The first numerical process makes it possible to detect 87 bead centres. From these points, the deformable surface algorithm was used to create 87 meshes corresponding to the repres entative volume of foam beads (figures 11 and 16a, b and c). This method was applied for all the acqu ired tomograms (points A, A', B, B'..., figure 3). It is then possible to represent the sample as a set of surfaces and better visualise its deformation during the experiment: figure 11 shows this representation before impact and after the second impact (respectively left and right view). Each mesh can then be extracted and its deformation, which approximated that of the corresponding bead, studied separately; in many cases, the bead 
strain is more complex than the strain ob tained under a simple homogeneous uniaxial compression. For instance, if we consider the bead marked as number 61 and shown in figure 12 , visual analysis for the two first impacts reveals a inhomogeneous deformation; the lower portion of the mesh seems to become more compressed than the rest [14].

This qualitative observation can be completed by quantitative measurement in using the features of numerical algorithm. Firstly, for each bead, voxels contained in the RBV are counted: this gives a lower bound of the bead volumes, since the volume of a voxel is $4.91^{3}$

$\mu^{3}$. The intersticial volume between beads can be quantified from the ratio between the volume of meshes and the one of the sample (which we suppose being a perfect cylinder). For the CT-scans before loading and after the first and second impacts, this ratio is close to 0.74 . For the third and fourth impacts (where the macroscopic strain reaches 50 and $70 \%$ ), this ratio is respectively 0.64 and 0.51 . It means that this algorithm is sufficien tly precise to obtain meshes with a nearly constant intersticial volume if the macroscopic deformation of the sample is lower than $50 \%$. For higher deformation rates, the foam structure becomes too complex (during its densification) and the volumes bounded by the meshes are less representative of the beads.

Secondly, an average grey level can be calculated for each bead, by using the intensities of the pixels of the tomograms that are contained in its corresponding mesh. With common image processing software, the average grey level of each RBV was calculated. Theoretically, there is a linear relation between the grey level of a pixel and the density of the corresponding volume element. Indeed, the to mographic reconstruction produces a $3 \mathrm{D}$ mapping of $\mu$, the linear attenuation coefficient of the material. The grey level image is obtained by applying the following affine transformation to this 3D mapping: the grey level assigned to the pixels having a value lower than $\mu_{\min }$ is 0 , for the pixels having a value larger than $\mu_{\max }$ it is 255 , and in between a linear interpolation is applied. The values of $\mu_{\min }$ and $\mu_{\max }$ are determined by the local range of porosity and the amount of noise in the acquisition. Noting that:

$$
\begin{aligned}
& \mu_{\text {air }} \ll \mu_{p p} \\
& \rho_{\text {air }} \ll \rho_{p p}
\end{aligned}
$$

the following relations can be obtained:

$$
\begin{aligned}
& \mu \cong(1-\phi) \mu_{p p} \\
& \rho \cong(1-\phi) \rho_{p p}
\end{aligned}
$$

where $\rho$ is the density, $\phi$ the porosity, and subscrip ts air and $p p$ refer respectively to air and polypropylene.

Combining equations 2 and the linear relation between $\mu$ and the grey level (between $\mu_{\min }$ and $\left.\mu_{\max }\right)$, poro sity and density could be obtained from grey level :

$$
\begin{gathered}
\phi_{i, j}=1-\frac{\mu_{i, j}}{\mu_{p p}} \\
\rho_{i, 0}=\frac{\mu_{i, 0}}{\mu_{p p}} \rho_{p p}
\end{gathered}
$$

With $i$ the bead number and $j$ the compression stage ( 0 before loading, 1 after the first impact, point $A$ in figure 3 , and so forth).

A volume variable can be chosen to estimate the deformation of each bead. If we consider the strain tensor $\overline{\bar{\varepsilon}}$, the trace of this tensor corresponds to the volume strain of the material $\varepsilon^{v o l}=\operatorname{tr}(\overline{\bar{\varepsilon}})$. The volume strain of a porous media can be calculated from its porosity. In our case, the volume strain of a bead $i$, due to the dynamic loading ( $\operatorname{step} j$ ), is obtained from the relation : 


$$
\varepsilon_{i, j}^{v o l}=1-\frac{\phi_{i, j}-\phi_{i, 0}}{1-\phi_{i, j}}
$$

It is then possible to retrieve the volume strain of each bead from the variation of its mean grey level in combining equations 3 and 4 :

$$
\varepsilon_{i, j}^{v o l}=\frac{\mu_{i, 0}-\mu_{i, j}}{\mu_{i, j}}
$$

\subsection{Foam morphology \\ a- Bead size}

For each bead, the number of pixels contained in the mesh multiplied by the corresponding volume occupied by a pixel $\left((4.91 \mu \mathrm{m})^{3}\right.$ in this case) approximately measures the RBV. This first calculation gives an indication on the bead size variability before the loading. Initially, the mean volume of the RVBs is $6.7 \mathrm{~mm}^{3}$, ranging from $0.8 \mathrm{~mm}^{3}$ to $12,9 \mathrm{~mm}^{3}$. The smallest RBVs correspond to the outer beads that have been cut during sample preparation. If we omit these incomplete beads, the mean volume rises to $9.7 \mathrm{~mm}^{3}$, ranging from $6.2 \mathrm{~mm}^{3}$ to 12,5 $\mathrm{mm}^{3}$. Assuming that there is a good correlation between the computed RBV and the corresponding bead in terms of volume, we observe a strong heterogeneity in bead size for the sample before impact. Paragraph 4.2 analyses the effect of this heterogeneity on bead deformation.

\section{$b$ - Bead density}

Theoretically, using equation 3 the average density of a bead can be obtained from the grey levels of all pixels contained in that bead. It can be approximated by using the pixels contained in the corresponding RBV. In practice, the average grey levels obtained for each bead varies only weakly, from 77 to 84 . The measurement dynamics, calculated as the ratio of grey level variation $(84-77)$ to the measurement range $(256)$, does not exceed $3 \%$. Those grey levels correspond to bead poro sities ranging from $98 \%$ to $78 \%$ (with an $89 \%$ average), and reveal for the first time a strong heterogeneity at the bead scale. Density heterogeneity also seems significant; the lower estimated densities are $10 \mathrm{~kg} / \mathrm{m}^{3}$ whereas the maximum values reach $200 \mathrm{~kg} / \mathrm{m}^{3}$. However, even if this high variation of porosity (i.e. density) is coherent with the visual observations, it is difficult to consider those results as realis tic since the noise associated with grey level measurements generates a significant error on local porosity.

Therefore, the grey levels were not used to determine bead densities with accuracy, but only to estimate relative bead density variations. In the rest of th is paper, the densities are provided only to give an indication of fo am density heterogeneity and should not be considered exact or absolute. It is then possible to plot the density distribution as a function of bead position. Recalling that the sample is cut in the middle of the polypropylene fo am plate to avoid any density gradient, we should not observe any relation between the two. If we consider bead position in the sample only in the vertical direction, the plot shown in figure 13a reveals a random distribution. On the contrary, if we consider bead position as distance from the cen tre axis of the cylindrical sample, depicted in figure 13b, then a strong density gradient is seen: the densest beads are located around the edges. The induced radial shear caused by the crown saw during sample cutting most probably increased the foam density in the periphery.

Further investigation of the volume strain of the beads will allow to evaluate the effects of this wide den sity distribution, a structural charactersistic of the sample, on local deformation.

\subsection{Volume strain}


The volume strain has been calculated (equation 5) for each RBV at different loading stages: before the impact, after the first impact when the sample is maintained compressed (point A figure 3), after the unloading of the first impact (point A' figure 3), etc. These results show what influences localisation of foam strain.

\section{a- Volume strain vs bead size}

The volume strain $\varepsilon_{i, j}^{v o l}$ of the beads has been evaluated at each step of the dynamic loading as a function of their size. Figure 14 shows the distribution of the volume strain vs the RBV values after the 4 first impacts when the sample is maintained compressed (points A, B, $\mathrm{C}$ and $\mathrm{D}$ in figure 3). For each step, the $\varepsilon_{i, j}^{\text {vol }}$ distribution is strongly dispersed. However, there is a slight trend that the bigger beads seem to be more deformed than the smaller ones. This trend is more visible after the second impact. In fact, the influence of the bead size is difficult to highlight. The existence of a correlation between bead size and density can be presumed from the moulding process: beads of nearly constant mass are injected in the mould and during expansion, those that are able to occupy a larger volume have a lower density. Therefore, the influence of bead size can be confirmed by analysing the effect of bead density on volume strain.

\section{b- Volume strain vs bead density}

The evolution of the volume strain has been established as a function of bead density for the 4 impacts when the sample is still compressed (figure 15a) and after unloading (figure $15 \mathrm{~b})$. The two configurations lead to the same remarks. For the first impact, the influence of the density on $\varepsilon_{i, j}^{v o l}$ can be detected despite a strong scattering. Coarsely, the lower the density, the greater the bead volume change. The effect of density is confirmed by the following loading stages, when the macroscopic strain imposed on the sample reaches high values. Lower den sity beads are siginificantly compressed whereas the denser ones -more rigid and resistant- are less deformed. This is in accordance with the fact that, firstly, the volume strain is a combination of elastic and plastic deformation and the values of $\varepsilon_{i, j}^{\text {vol }}$ depend on bead density, and secondly, when the sample is unloaded, $\varepsilon_{i, j}^{\text {vol }}$ which corresponds to the plastic or residual deformation also depends on this parameter.

To conclude on the influence of the foam morphology on its mesoscopic volume strain, we have und erlined a sign ificant correlation between bead volume change and density (and size by duality). The previous remarks appear to be evident, as many studies have already shown the influence of the foam density on its macroscopic behaviour: typically, denser foam has a higher mech anical response. However, to our knowledge, it is the first time that under dynamic loading and at the mesoscopic scale, the influence of local density on bead deformation is shown. To complete this study and qualify the strain - density correlation, localisation of foam strain has been analysed.

\section{c- Volume strain vs bead position}

For each impact, bead volume strains were plotted versus barycentre positions; the objective is to detect any localisation of the strain at the scale of the sample. The coordinates of the bead barycentres are defined in a cylindrical system $(r, \theta, Z)$ since the sample is axisymetric. Given that $\theta$ has no influence (this hypoth esis has been checked), the beads (identified by a number, figure 16) are classified accord ing to the vertical and radial positions, $Z$ and $r$ respectively, of their barycentres. For the sake of clarity, the results are separated, firstly for Z, in five $2 \mathrm{~mm}$ high horizontal sections of the sample, and secondly for $\mathrm{r}$, in two cylindrical regions of identical volume: a central cylinder $(3.53 \mathrm{~mm}$ in diameter) and its 
oulying tube. Each bead is then assigned a horizontal section and a cylindrical region. For each defined portion of the sample, the average bead volume strain is calculated, and th is process is repeated for each impact.

The dependence of the strain $\varepsilon_{i, j}^{v o l}$ on the $Z$ position is illustrated in figure 17. Each diagram presents the average volume strain of the RBVs belonging to the five vertical sections for each impact and when the sample is still compressed or unloaded. Slice 1 corresponds to the bottom of the sample and slice 5 to the top of the sample, in contact with the compression punch.

For the first impact, the distribution of volume strain ac cording to $Z$ is clearly established: the slices closer to the compression surfaces of punch and die are more deformed than average (the global strain is $10 \%$ ) whereas the centre slice is only lightly deformed (5\%). This is verified in both the compressed and unloaded states. Moreover, the most deformed slice (number 5) is the one against the punch that inflicts the loading. Visual inspection of bead and cell wall deformation corroborates this analys is. Sev eral factors could explain this behaviour, and they can be associated to the type of compression and to the morphology of the structure. Firstly, concerning the compression procedure, the contact of the sample with the rigid surfaces of the punch and die can generate a greater deformation for border beads, especially during a dynamic loading; the punch applies a shock wave directly to slice 5 . There is not necessarily an equilibrium of the forces applied on the sample at the beginning of the test (th is phenomenon has been proved in the case of dynamic loading of cellular materials with a Hopkinson Bar). In this case, at the beginning of the test, the compression force is concentrated on slice 5 and generates a higher damage. Secondly, and this concerns the preparation of the sample, the outer beads in contact with the punch and die have been cut and are thus weakened by the loss of part of their walls. The morphology of the foam structure is different in these two zones and can contribute to the modification of the behaviour.

For the second impact, the observed phenomena are similar. The global deformation imposed on the sample is $30 \%$. In the compressed state, the higher slices are the most deformed: the volume strain of slice 5 reaches $38 \%$ whereas slice 2 is just deformed by $20 \%$. Again, the beads in contact with the rigid surfaces of the die and punch are particularly deformed. It is also interesting to compare the volume strain in the compressed and unloaded states. When the sample is unloaded, the measured volume strain corresponds to the residual or "plastic" deformation. In this configuration, the residual deformation is nearly constant for slices 2,3 and 4 after the second impact ( $\varepsilon_{i, 2}^{v o l}=20 \%$ ), but in its compressed state, a strong difference can be noted. This means that the centre of the sample is not irreversibly damaged; the residual volume strain is low (less than $20 \%$ ) and the elastic response is still significant for slices 3 and 4 . On the contrary, the elastic response is particularly weak for slices 1 and 5 whereas the strain $\varepsilon_{i, 2}^{v o l}$ is significant. It seems that damage to the beads located in these two sections is high. This state of the microstructure has been controlled by visualising the shape of bead and cell walls.

For the two last impacts corresponding respectively to a deformation of 50 and $70 \%, \varepsilon_{i, j}^{v o l}$ is more homogeneous; a slope is observed in the evolution of the volume strain accord ing to $Z$; the denser slice (number 5) is again the one aga inst the compression punch. Furthermore, for these levels of compression, the cellular material becomes strongly damaged (the sample is in the densification phase after the 4th impact), deformation of slice 5 reaching $71 \%$. By the end, the elastic response (defined as the difference between the strains measured when the sample is compressed and unloaded) is very low and the values of volume strain are high.

We have shown a dependence of the volume strain $\varepsilon_{i, j}^{v o l}$ of the beads on their vertical position $Z$, after each impact. At the beginning, only the region s close to the punches are damaged, while the centre beads bear little strain. After more impacts, th is heterogeneity decreases and 
damage spreads to all slices. Finally, the residual or plastic deformation increases for each region whereas it has been shown that the elastic response tends to zero after all impacts. The heterogeneity of $\varepsilon_{i, j}^{v o l}$ and its evolution depends on the combination of the effect of the dynamic loading (certainly due to a propagation of a shock wave during the impact) and the effect of the foam microstructure. The beads cut during the preparation of the sample are more fragile and are the first to become damaged. The modification of the microstructure (some bead walls have been removed) has a strong consequence on the foam behaviour at the small scales (bead and cell scales) even if the macroscopic behaviour is unaltered - the response of the polypropylene foam measured during the impact corresponds to a typical behaviour for a cellular material; an elastic response followed by a plastic plateau and finally a densification [8].

Finally, bead volume strain is compared to radial position in table 1 . Values of $\varepsilon_{i, j}^{v o l}$ in the periphery are lower than those in the central region after each impact and whether the sample is compressed or unloaded. This variation in strain according to radial position is certainly due to two phenomena. Firstly, it has been shown that for this sample there is a dependence of the density on the radial position. Subsequently, this strain heterogeneity is one effect of the gradient of bead density. Secondly, the denser peripheral beads constitute a rigid shell for the core of the sample. During the compression, the volume variation of this external shell skin is low, and its thickness increases while its height decreases. The increase in thickness implies a radial stress imposed on the central beads. For these beads, the loading is in fact a combination of an uniaxial compression and a radial pressure: their volume strain is higher. Therefore, this particular heterogeneous microstructure in terms of bead density gen erates a stress field between beads more complex than the uniaxial compression imposed by the punch. This hypothesis is confirmed when observing the shape of the deformed beads [15].

\section{d-Conclusion}

This analysis identifies the effect of different parameters on the deformation of the cellular material at the scale of the beads. Influence of parameters such as bead size and density has been evaluated and localisation of mesoscopic deformation is highlighted. Many publications have already demonstrated the influence of foam density on mech anical response at the macroscopic scale, but this original approach allows to characterise at a finer scale and during dynamic loading the effects of bead density on volume strain. Density may have an influence on local deformation in different ways. First, we have shown that denser beads are less deformed, but this microstructural heterogeneity in troduces a specific mesoscopic behaviour: the force field generated during impact depends on the geometry of the microstructure and bead density distribution. The influence of bead walls was also described. During sample preparation, the microstructure of the cellular material was modified in that the peripheral beads cut in the process were weakened. The thicker bead walls then influence the behaviour of the cellular material.

In order to confirm that density is not the only parameter that acts on sample deformation, volume strain was examined for specific beads. Four beads have been selected in the centre of the sample since deformation in this area seems more homogeneous. Figures 16 allows to visualise the positions and shapes of these beads. Table 2 indicates the bead number and density, and gives the volume strains calculated for the three last impacts (the results for the first impact are not significant). When comparing the volume strain calculated for beads 31 and 41 (of equal density) after the second impact, one observes that their deformation is clearly different. Similarly, volume strain of bead 45 is higher than the one of bead 61 whereas their densities are equivalent. On the other hand, between beads 61 and 31, 
deformation is similar but bead 31 has a higher density. These observations can be made for other beads at other levels of the impact (what level ? position?).

Consequently, it is clear that bead density has an influence on local deformation but the morphology of the structure (size and shape of the beads, thickness of bead walls...) must also be taken into account to improve constitutive model of the of this multiscale material.

\section{General conclusion and perspectives}

This articles presents the last results on the analysis of the localization of foam strain at mesoscopic scale. To reach these results and add a complementary description of the damage phenomena of a polypropylene foam under dynamic loading, a new approach has been developed in combining original impact device, micro tomography techniques and specific image algorythms. Firstly, dynamic compression interrupted tests were carried out on polypropylene foam and micro tomographic acquisitions (made after each impact) allowed visualising bead wall buckling within the sample. First analysis of the reconstruction in 2D obtained from these acquisitions have shown the heterogeneity of the strain at the bead scale. These encouraging results, presented in a previous paper, have to be confirmed by the study of the strain field ( at the mesoscopic scale) on the entire impacted sample. It was then necessary to developp new numerical algorithm to extract the volume of each bead from the complex microstructure of the multi scale foam. An image processing method has been then applied to extract pertinent information from the microtomograms and a representative volumes of foam beads have been underlined in 3D. The evolution of these meshed deformable surfaces allows determining the porosity variation of beads and their volume strain at several steps of the dynamic compression. A map of volume strain was therefore established and compared with initial density field.

Influence of parameters such as bead size and density has been evaluated and localisation of mesoscopic strain is highlighted. This approach has also allowed characterising at a finer scale and during dynamic loading the effects of bead density on the volume strain. The denser bead, the lower volume change. Nevertheless, density is not the only parameter that acts on sample deformation, the thicker bead walls also influence the behaviour of the cellular material. Moreover, it was shown that the combination of the microstructure and bead density distribution has an influence of the volume strain field. The analysis of these data concerning specific beads have revealed that bead density and volume change are not necessarily correlated. A complementary study using our micro tomo grams and 3D Digital Image Correlation techniques [14] had confirmed our measurements of the mean volume change on a bead (labelled 61) and had furthermore high lighted the heterogeneity of strain inside this bead, at a scale close to the cell scale

To conclude, bead density has an influence on local strain but the morphology of the structure (size and shape of the beads, thickness of bead walls...) has to be also taken into account to improve behaviour model of the of this multi scale cellular material. From this analysis, the behaviour of this EPP foam is better understood at mesoscopic scale. The next step of this research is the modelling of the foam morphology (bead and cell structures) taking into account the phenomena observed. A multi scale model will be soon developed representing the dense wall of beads (mesoscopic scale) by a surface mesh (with properties of dense polypropylene) and volumic meshes corresponding to the volume of cells within beads.

The next challenge of this study will be to construct this finite element model from the extracted cellular structure and verify model accuracy by comparing its numerical results with experimental ones. 


\section{Bibliography}

[1] Luca Di Landro, Giuseppe Sala and Daniela Olivieri, Deformation mechan isms and energy absorption of polystyrene foams for protective helmets, Polymer Testing, 2002, vol. 21(2), p.217-228

[2] M. Avalle, G. Belingardi and R. Montanini, Characterization of polymeric structural foams under compressive impact loading by means of energy-absorption diagram, Interna tional journal of impact engineering, 2001, vol. 25(5), p. 455-472

[3] Jun Zhang, Noboru Kikuchi, Victor Li, Albert Yee and Guy Nusholtz, Constitutive modeling ofpolymeric foam material subjected to dynamic crash loading, - International journal of impact engineering, 1998, vol. 21(5), p. 369-386

[4] J. Zhang, Z. Lin, A. Wong, N Kikuchi, V.C. Li, A.F. Yee and G.S. Nusholtz, Constitutive modeling and material characterization of polymeric foams, In ternational journal of engineering materials and technology, 1997, vol. 119(3), p. 284-291

[5] Bo Song, Weinong W. Chen, Songbai Dou, Nancy A. Winfree and Joseph H. Kang, Strain-rate effects on elastic and early cell-collapse responses of a polystyrene foam, International journal of impact engineering, 2005, vol. 31(5), p. 509-521

[6] Ph. Viot, F. Beani, and J-L. Lataillade. Polymeric Foam behavior under dynamic compressive loading. International Joumal of Materials Science, 2005. volume 40, number 22, pp 5829-5837. DOI 10.1007/s 10853005-4998-5

[7] Ph. V iot and P. Vacher. Identification of foam behavior under dynamic loading by the us e of partic le imaging techniques. Revue Matériaux et Techniques, hors série:39-43, December 2004. ISS N 0032-6895.

[8] Philippe Viot, Dominique Bernard and Erwan Plougonven, Phenomenological study of polymeric foam deformation under dynamic loading by the use of microtomographic technique, Joumal of Materials Science, to be published

[9] Peter Cloetens, Raymond Barrett, José Baruchel, Jean-Pierre Guigay and Michel Schlenker, Phase objects in synchrotron radiation hard x-ray imaging, Journal of phys ics D: applied physics, 1996, vol. 29(1), p. 133-146

[10] T.Y. Kong and A. Rosenfeld, Digital topology: in troduction and survey, Computer vision, graphics and image processing, 1989, vol. 48(3), p. 357-393

[11] Henk J. Heijmans, Mathematical morphology: a modern approach in image processing based on algebra and geometry, SIAM Review, 1995, vol. 37(1), p.1-36

[12] Hervé Delingette, Modélisation, déformation et reconnaissance d'objets tridimensionnels à l'aide de maillages simplexes, $\mathrm{PhD}$ thes is, Ecole centrale de Paris, 1994

[13] Johan Montagnat, Hervé Delingette, Nicolas Scapel and Nicholas Ayache, Representation, shape topology and evolution of deformables surfaces. Application to $3 D$ medical image segmentation, Research report $n^{\circ} 3954$, INRIA, 2000

[14] Stephane Roux, François Hild, Philippe Viot and Dominique Bernard, Three dimensional image correla tion from X-Ray computed tomography of solid foam, [submited for publication], 2007

[15] P. V iot, D. Bernard et E. Plougonven, Développement d'une méthodologie pour l'étude des déformations de mouss e polymère sous compress ion dynamique -Utilis ation de la microtomographie-Revue des Composites et des Matériaux Avancés, vol 17 (n¹) pp 9 23, 2007. ISBN 978-2-7462-1772-0. 


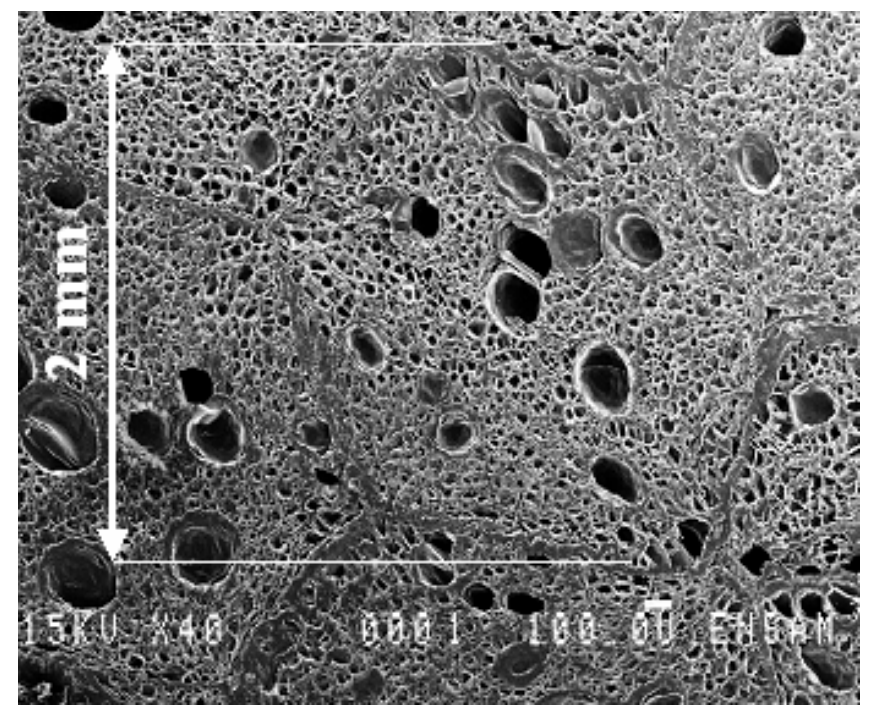

Figure 1a: Micrograph of polypropylene foam showing the bead structure

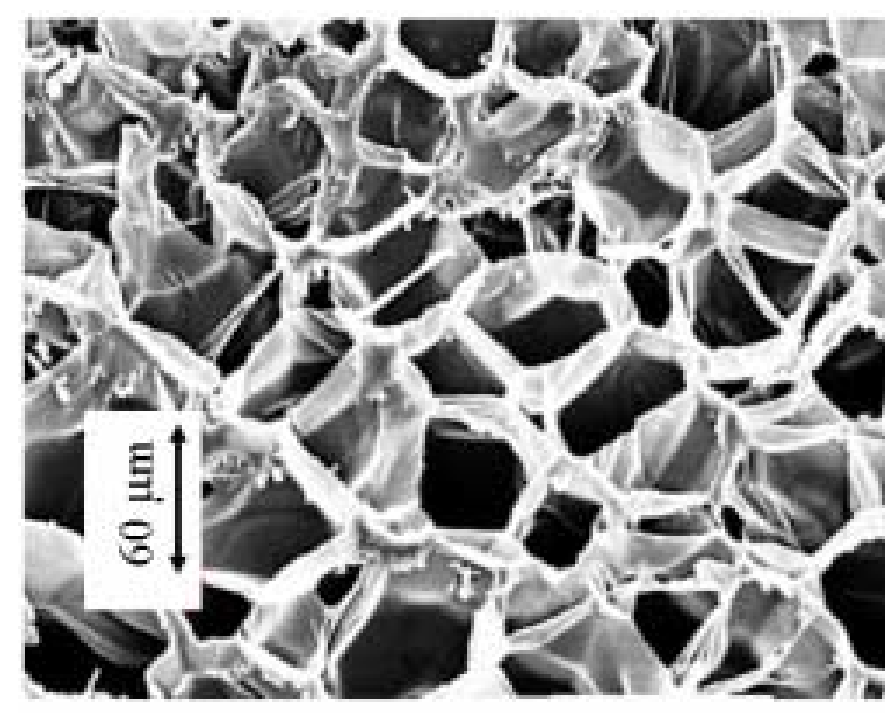

Figure 1b: Micrograph of polypropylene foam showing the cell structure 


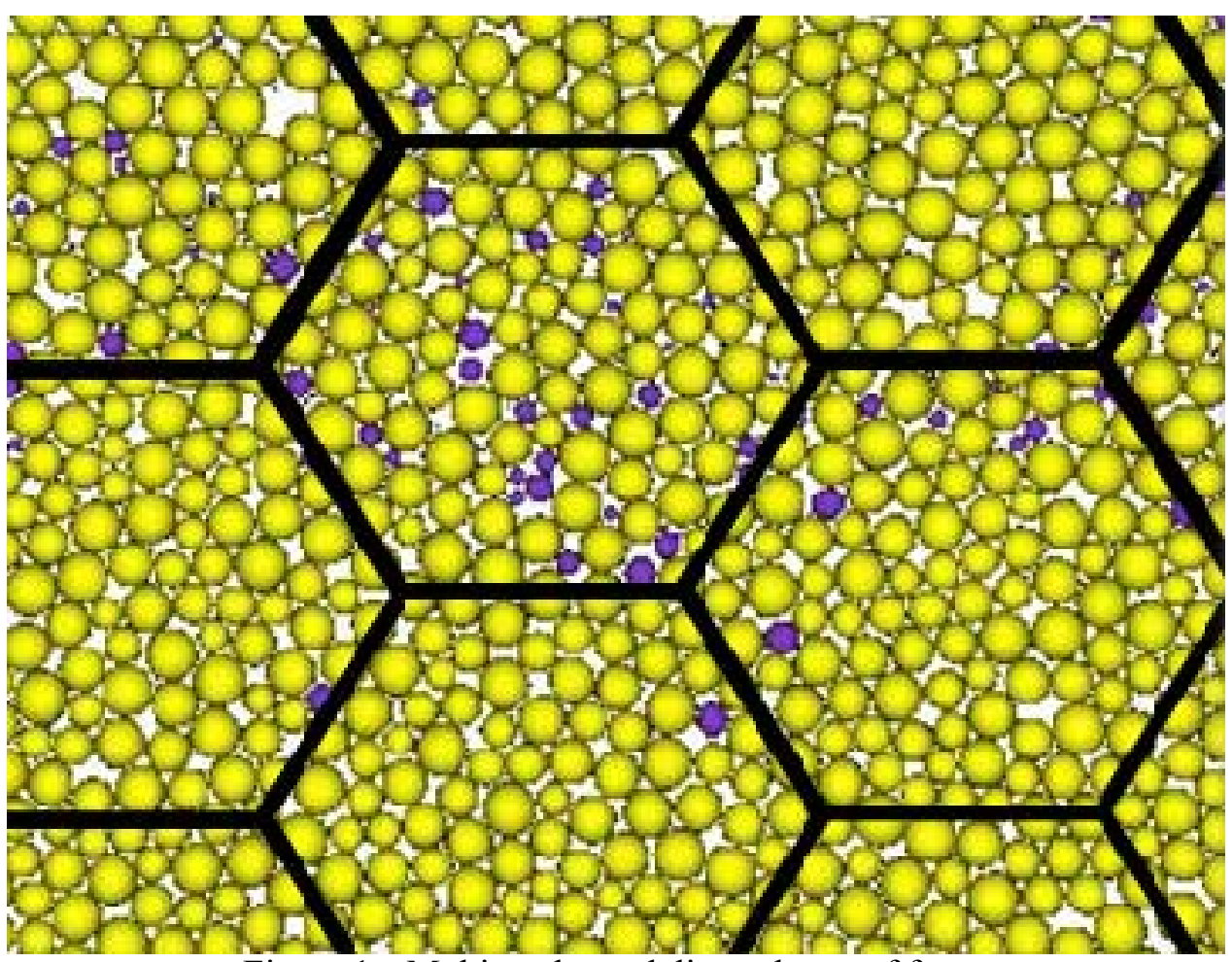

Figure 1c: Multi-scale modeling scheme of foam

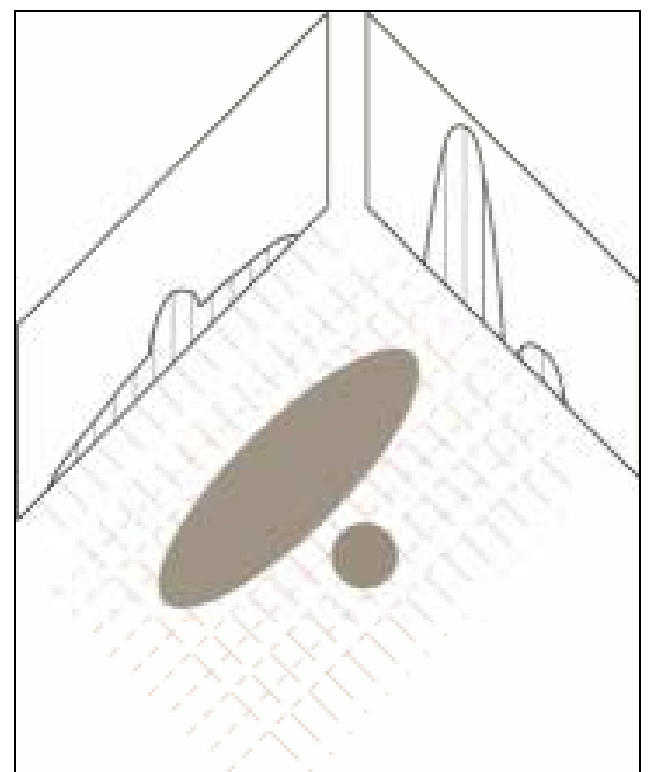

Figure 2: Illustration of the principle of parallel beam X-ray tomography 


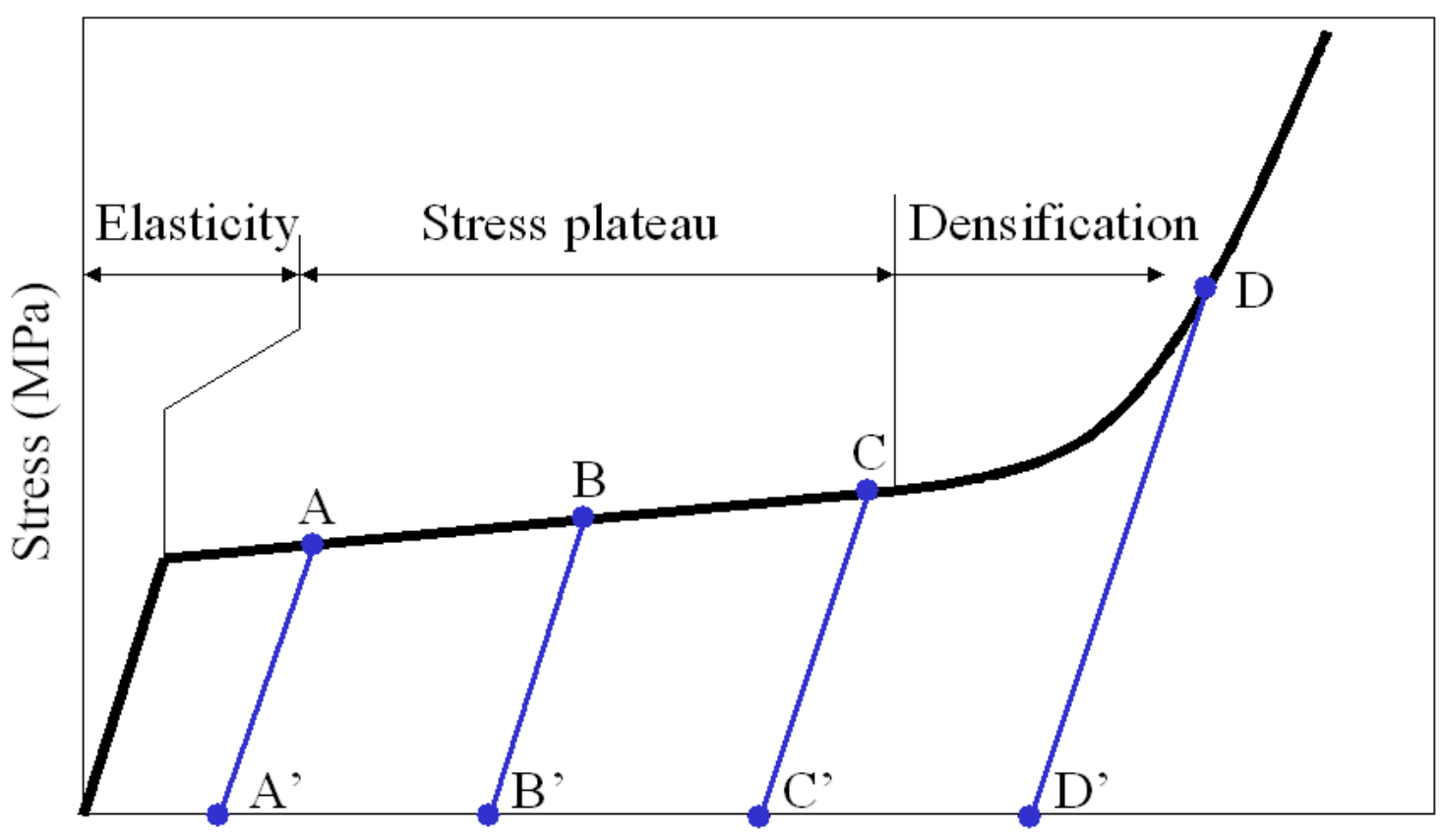

\section{Displacement $(\mathrm{mm})$}

Figure 3: Typical evolution of compression stress versus strain for a cellular material

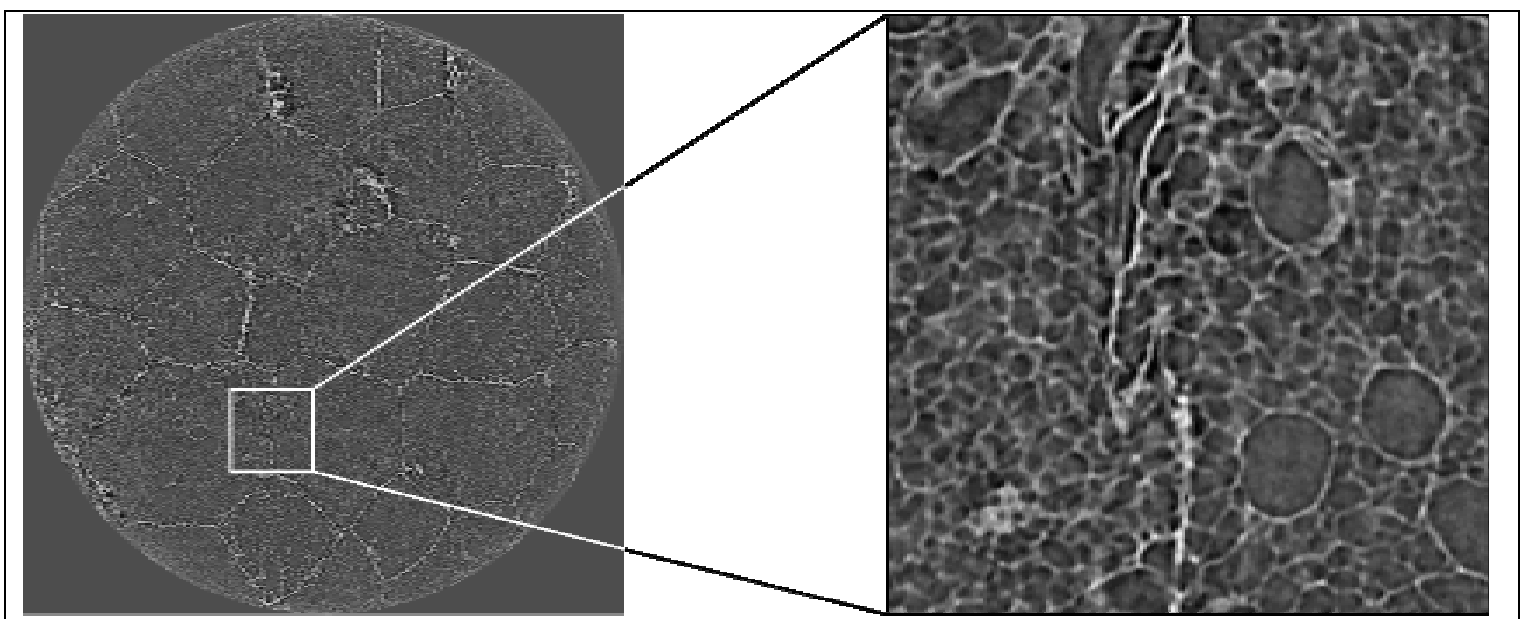

Figure 4: An axial cross-section of a microtomogram of the studied polypropylene foam. The left image is a view of the entire section, where the bead walls seem well-defined. The image on the right is a zoomed portion of the image, an example of why localising these walls with precision is not possible. 


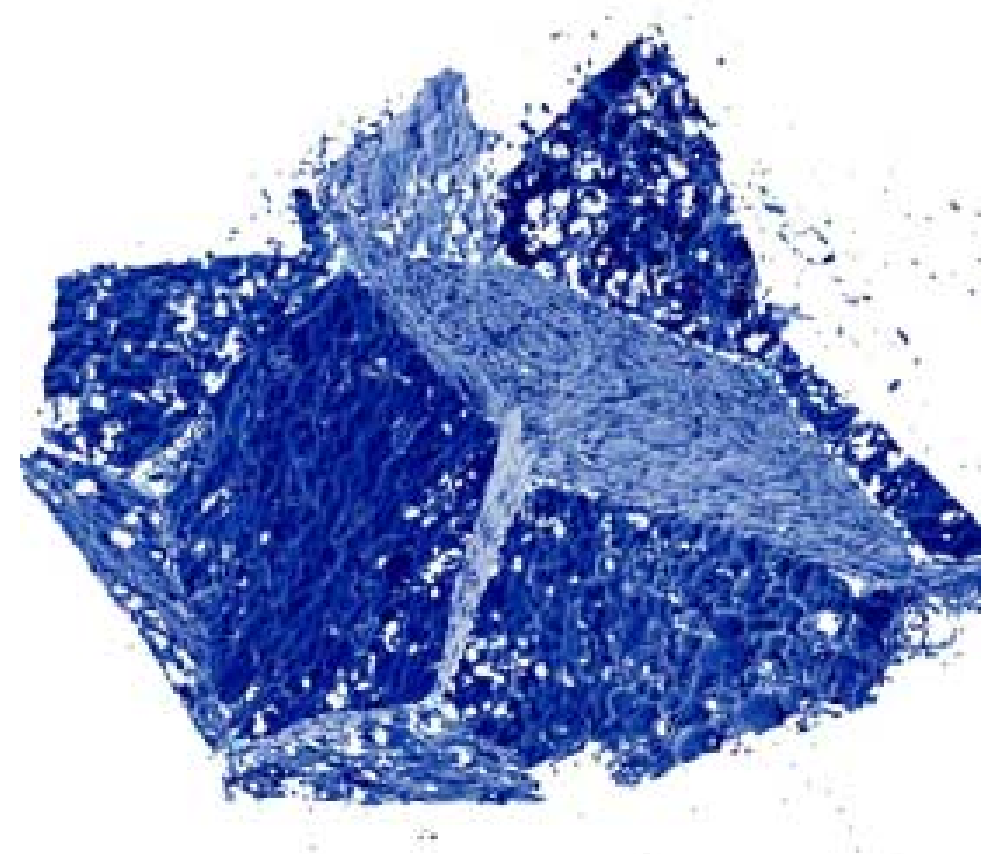

Figure 5: 3D reconstruction of bead walls in using classical numerical filters 


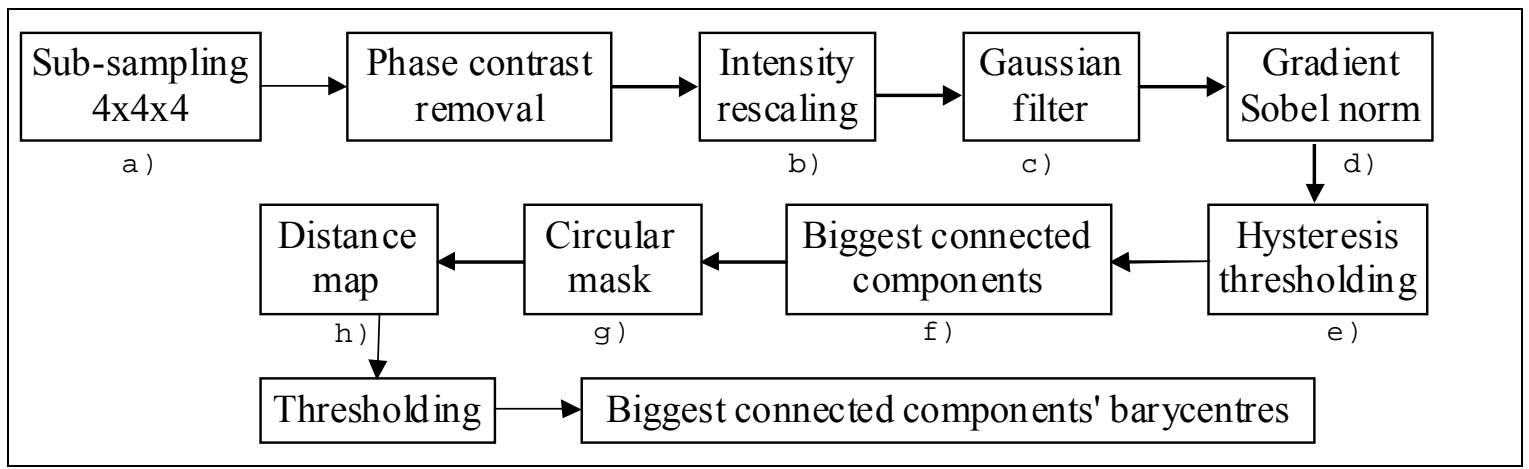

Figure 6: Method applied to the reconstructed microtomograms in order to extract approximate grain centres. Some parameters (such as the theshold levels) need to be manually set. Illustrations at steps a) through $\mathrm{h}$ ) are presented in figure 7.

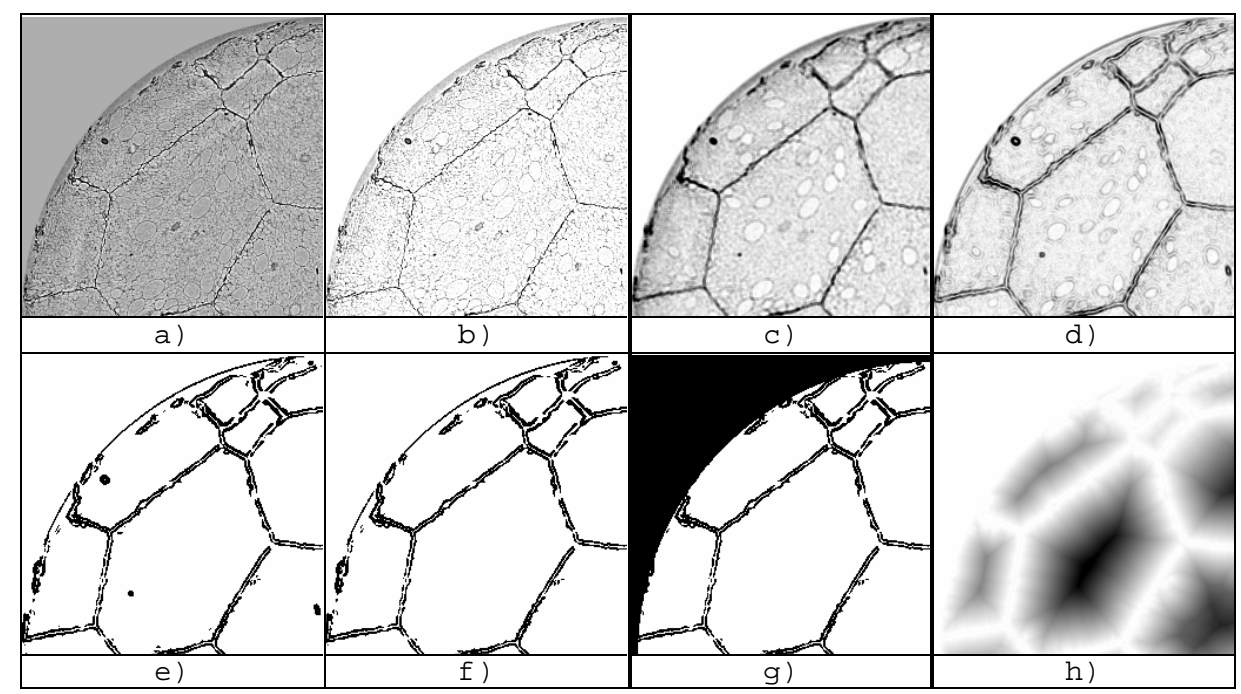

Figure 7: Illustration of the method used to extract approximate positions of the grain centres. It consists of a series of simple arithmetic and morphological operators on the microtomograms. 


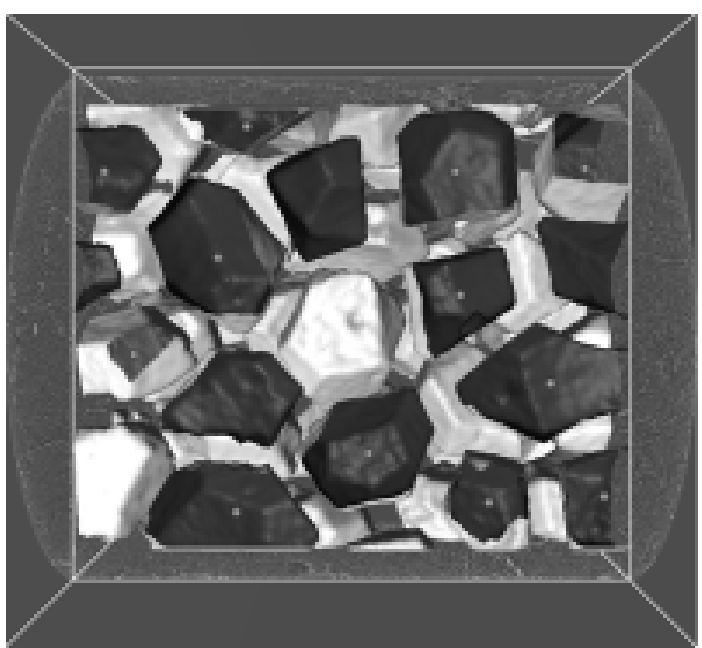

Figure 8: Representation of the distance map thresholding which allows to locate bead centres. An isosurface of the distance map (figure $7 \mathrm{~h}$ ), with the threshold value used for finding the barycentres, is displayed. The dots inside the isosurfaces are the bead centres.
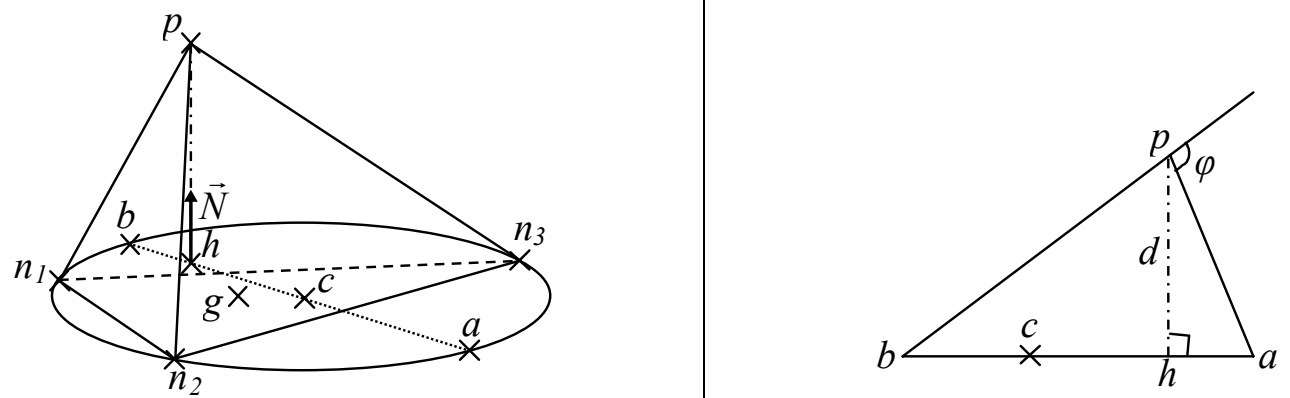

Figure 9. Simplex angle and elevation of a vertex of the deformable mesh, used to regularise the mesh to obtain a smooth surface. $p$ is the considered vertex, $n_{1}, n_{2}$ and $n_{3}$ are its neighbours, $h$ is $p$ 's projection on the plane defined by the triangle $n_{1} n_{2} n_{3}, c$ is the centre of the circle circumscribing $n_{1} n_{2} n_{3}, a$ and $b$ are the points of the circle aligned with $(h c)$, and $g$ is the triangle's centre of gravity. Finally, $d$ is called the elevation and $\varphi$ the simplex angle of $p$. 


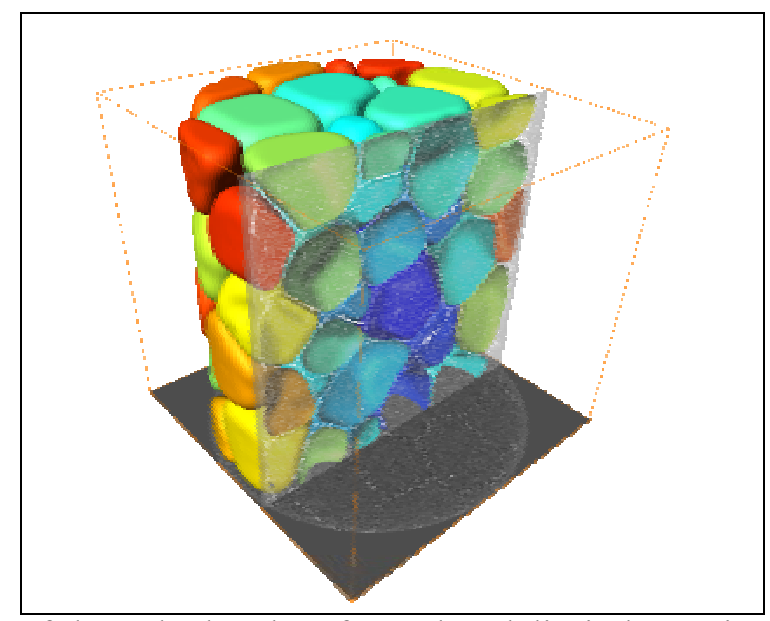

Figure 10: A view of the calculated surfaces that delimit the grains, with a colourmap corresponding to the relative density of the beads. Beads at the periphery are noticeably denser.

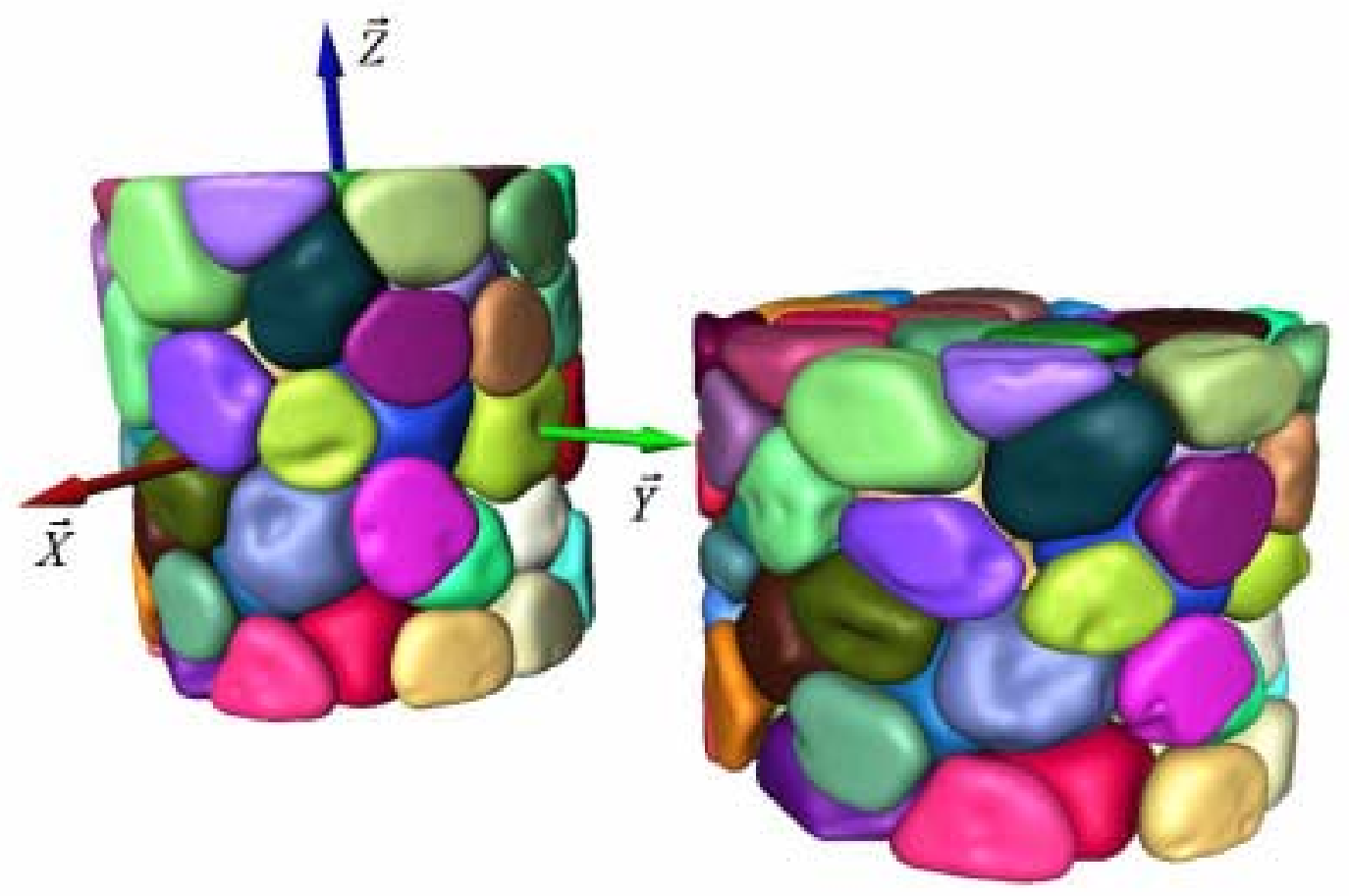

Figure 11: Reconstruction of bead morphology: before compression (left picture) and after the second impact (right picture). Each bead is identified by its colour. 

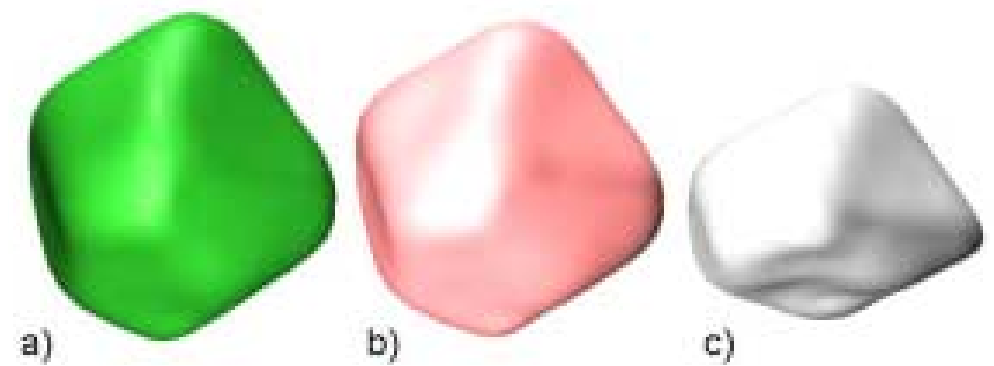

Figure 12: Reconstruction of the morphology of bead 61: before compression (a), after the first impact (b) and after the second one (c)

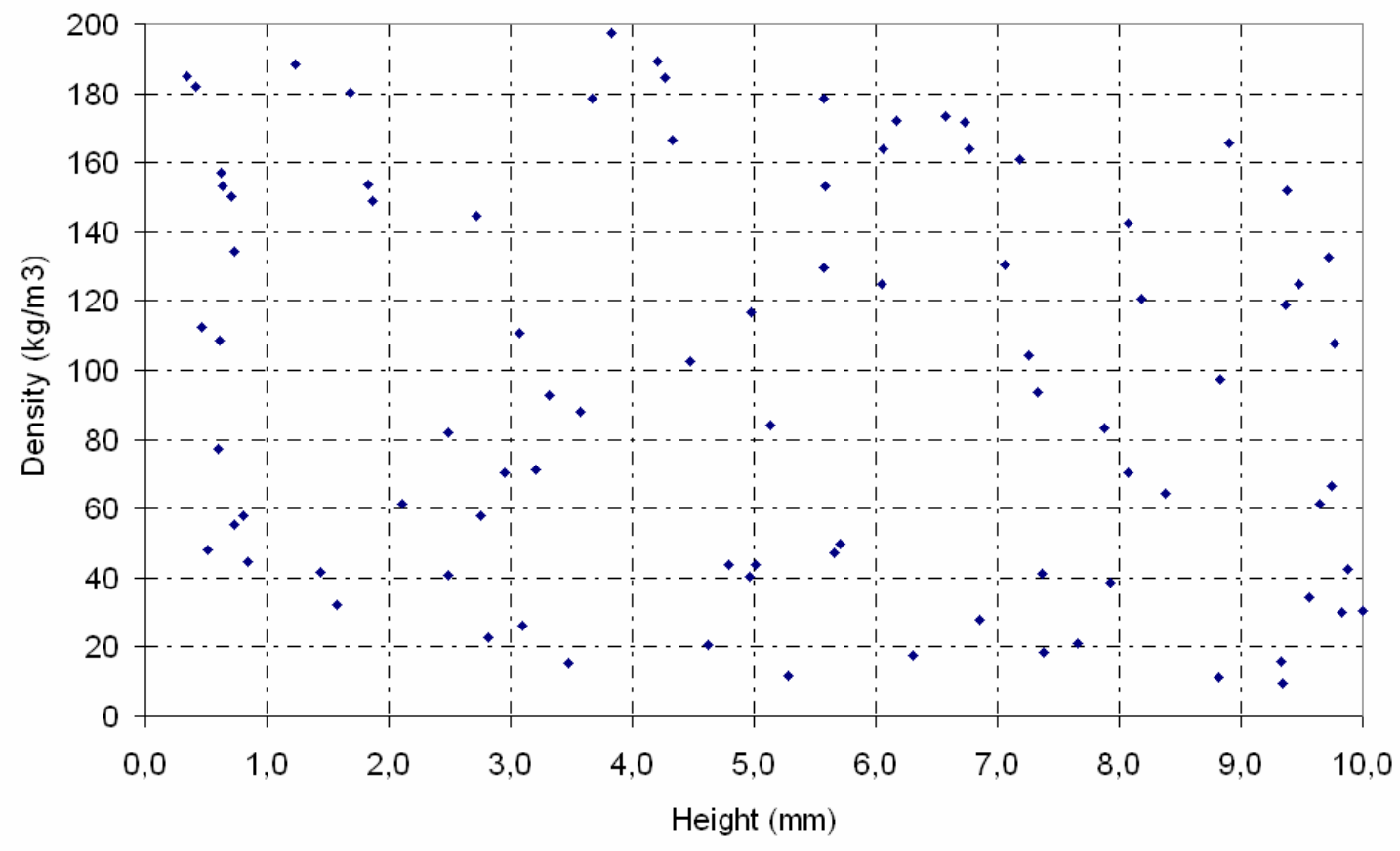

Figure 13a: Bead density as a function of vertical position in the sample 


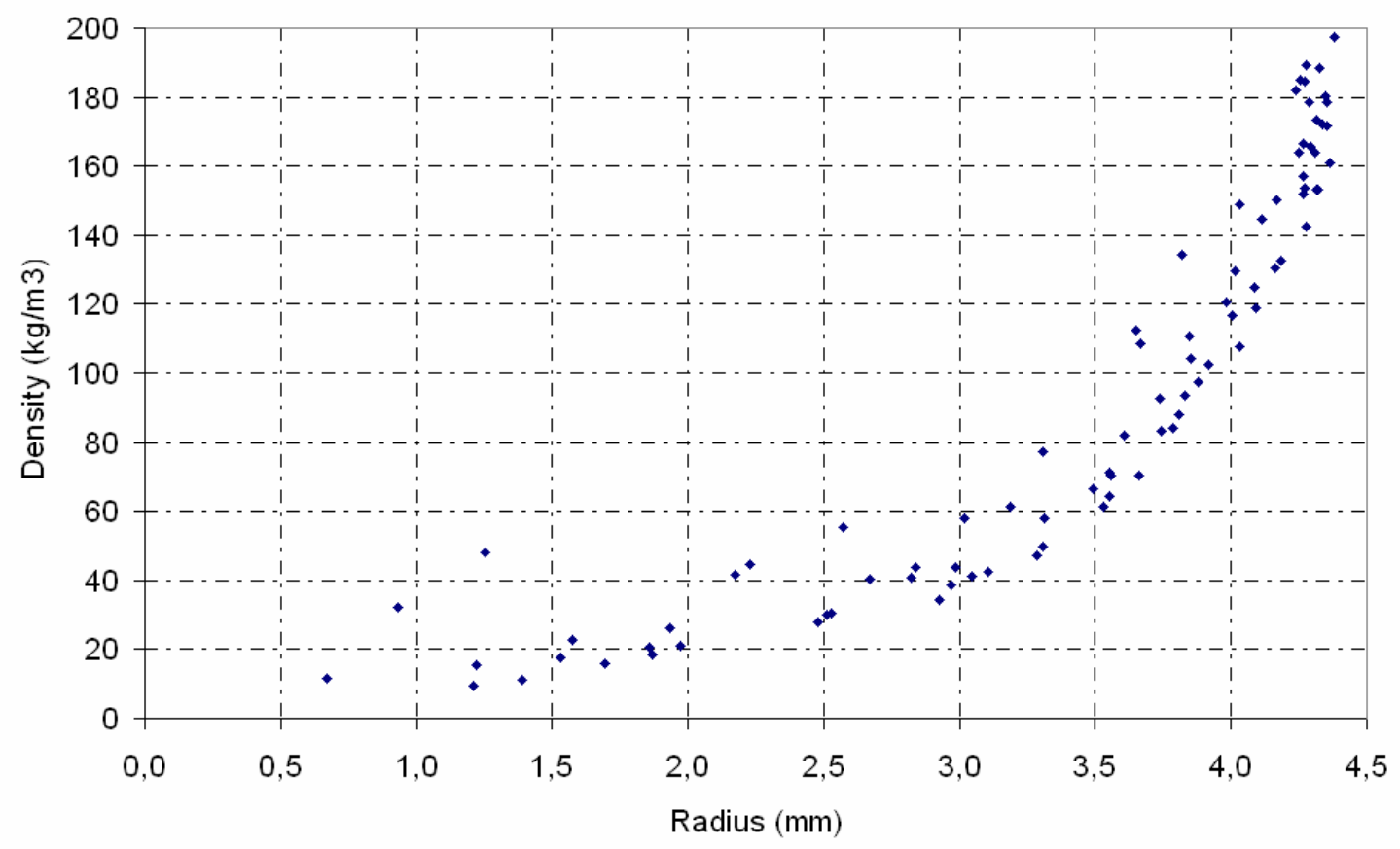

Figure 13b: Bead den sity as a function of its radial position

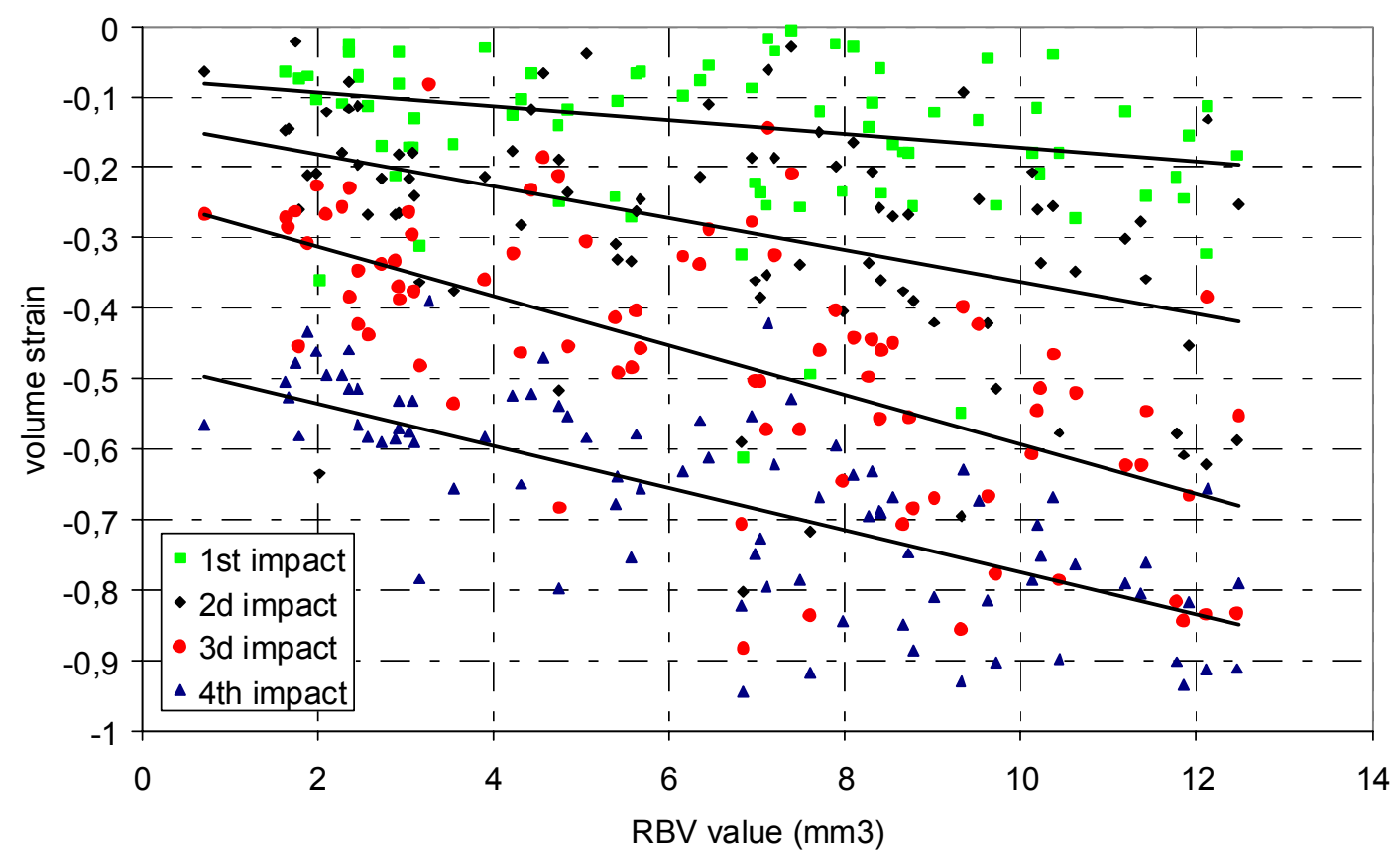

Figure 14: RBV values as a function of volume strains, plotted for each bead and at each impact stage 


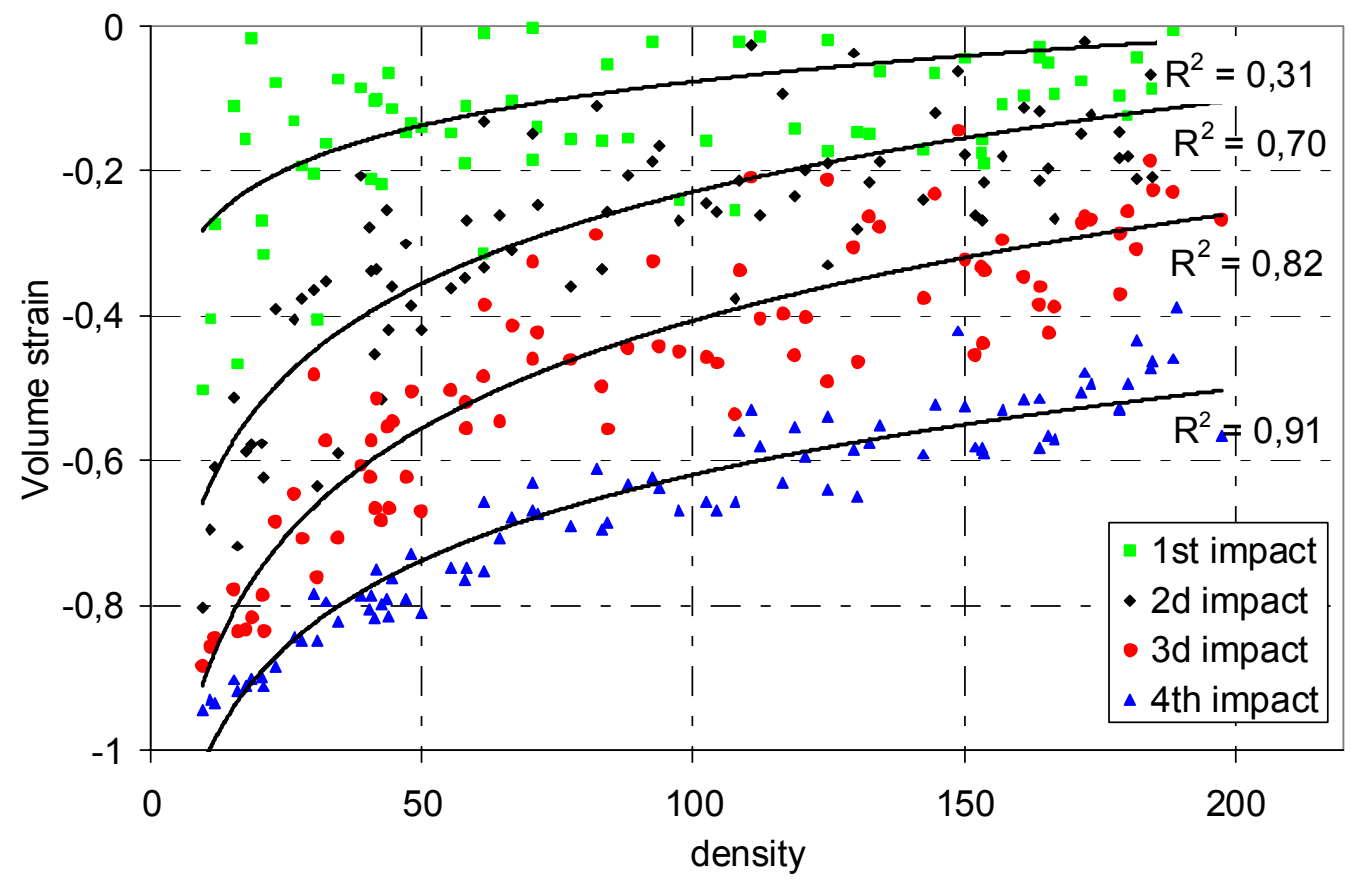

Figure $15 \mathrm{a}$ : Bead density as a function of RBV variation after each impact when the sample is maintained compressed

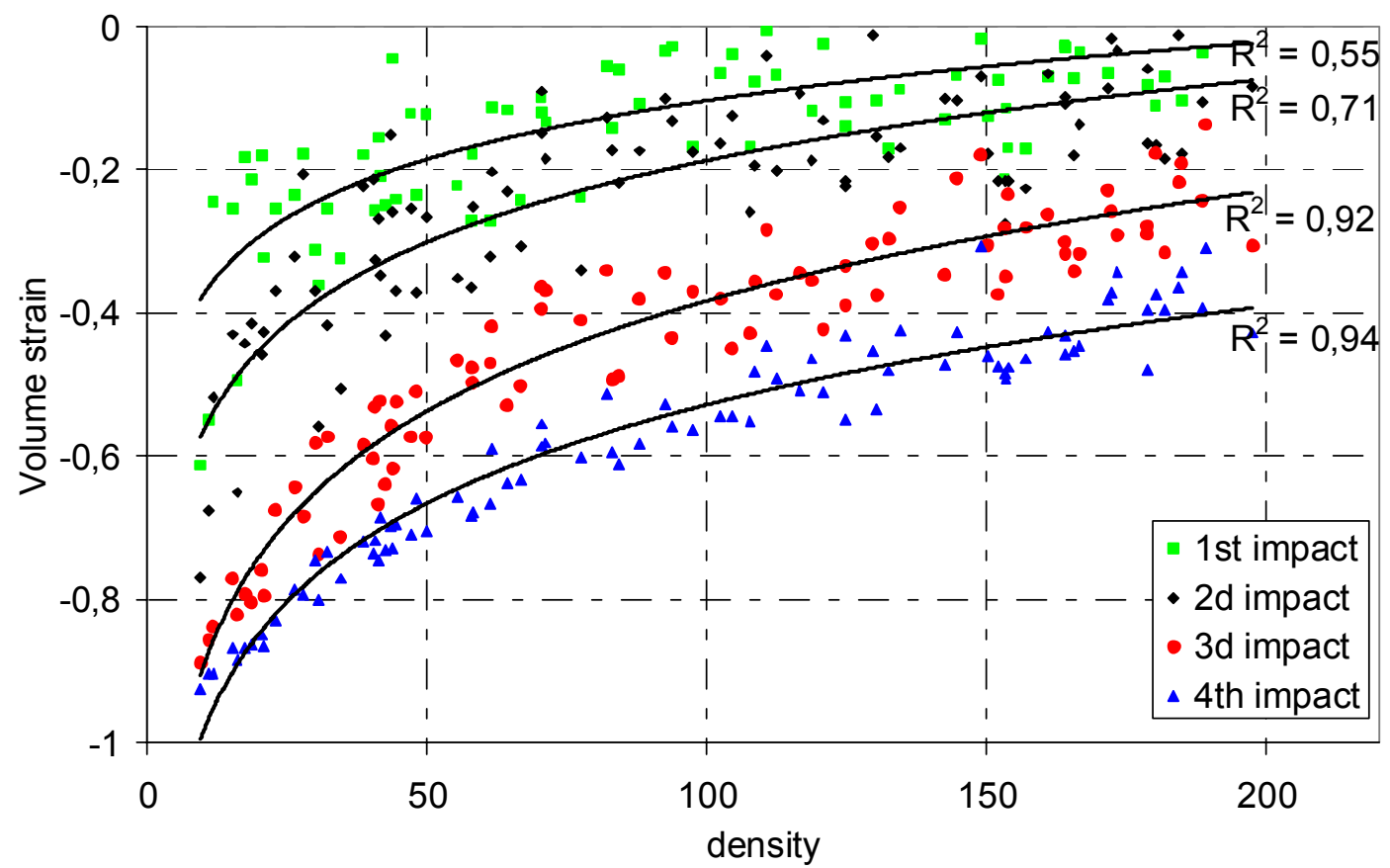

Figure 15b: Bead density as a function of RBV variation after each unloading stage 

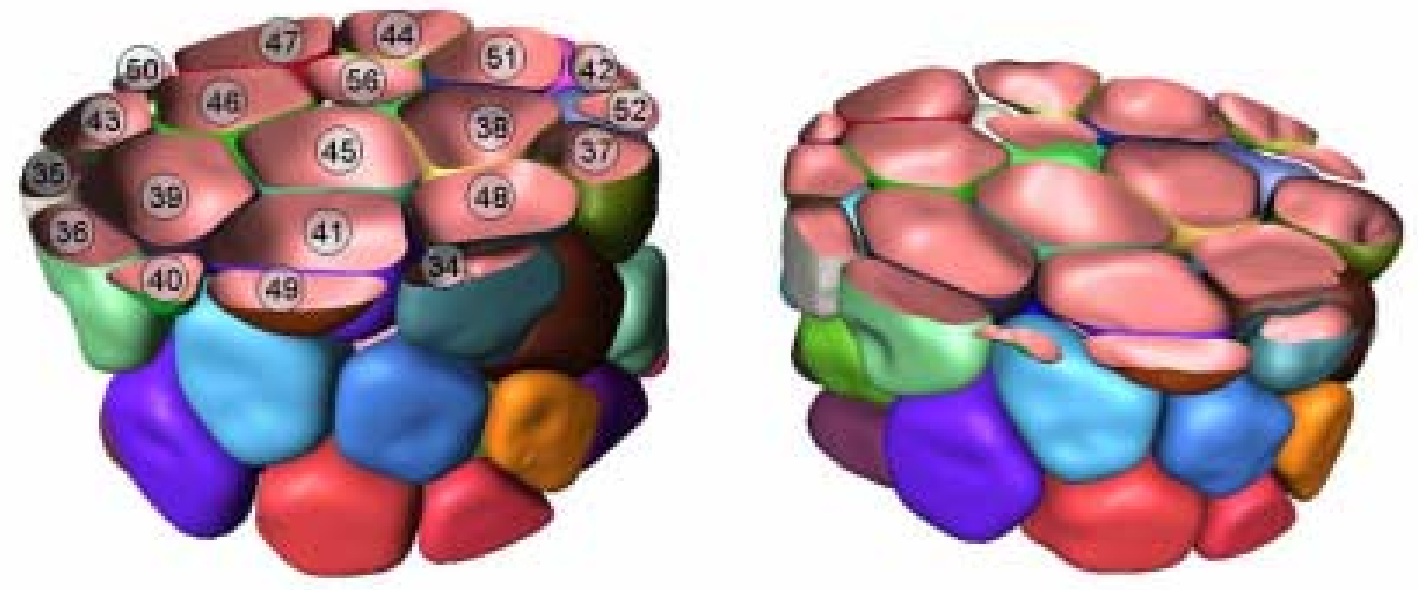

Figure 16a
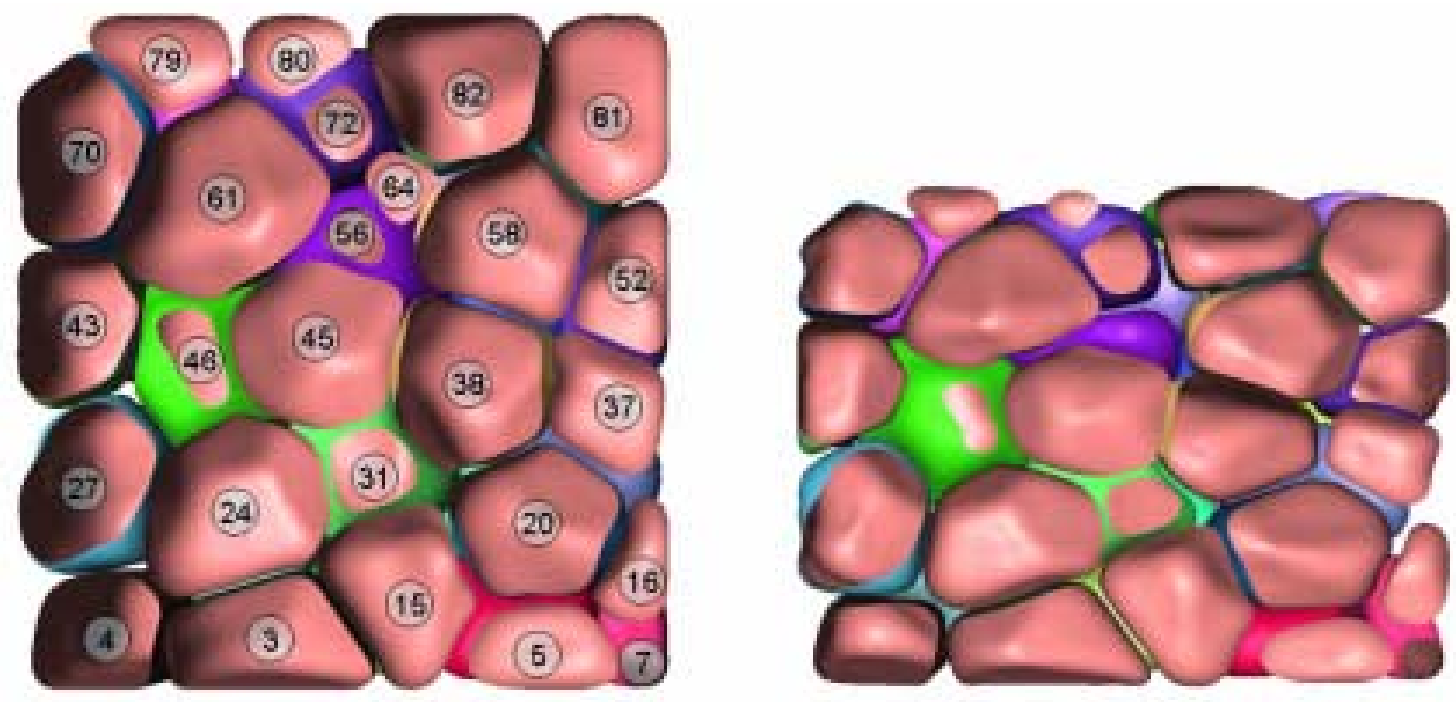

Figure 16b
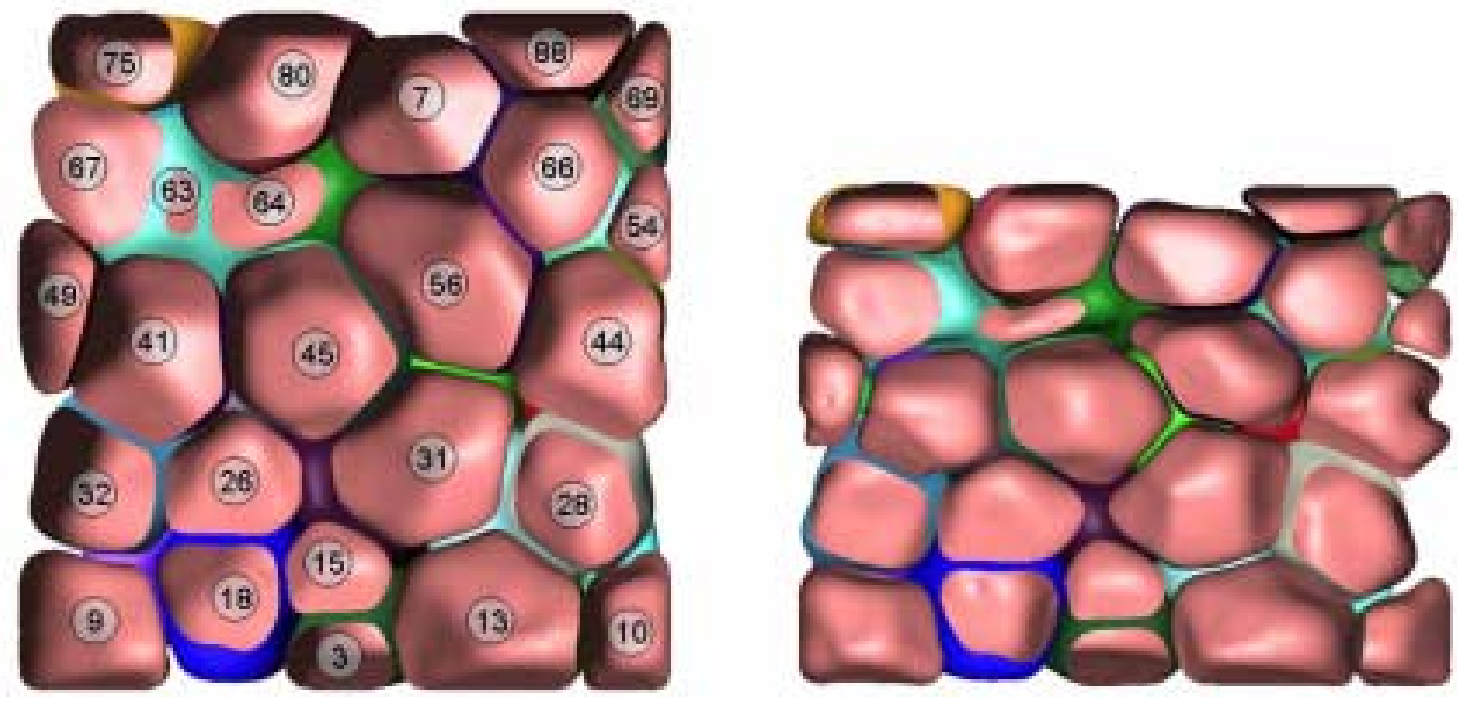
Figure $16 \mathrm{c}$

Figure 16: Slices in different directions (a: XY or axial, b: XZ or coronal, c: YZ or sagittal) of the RBVs for two stages: before impact (left), and after the second impact (right). Beads are all identified by a number.
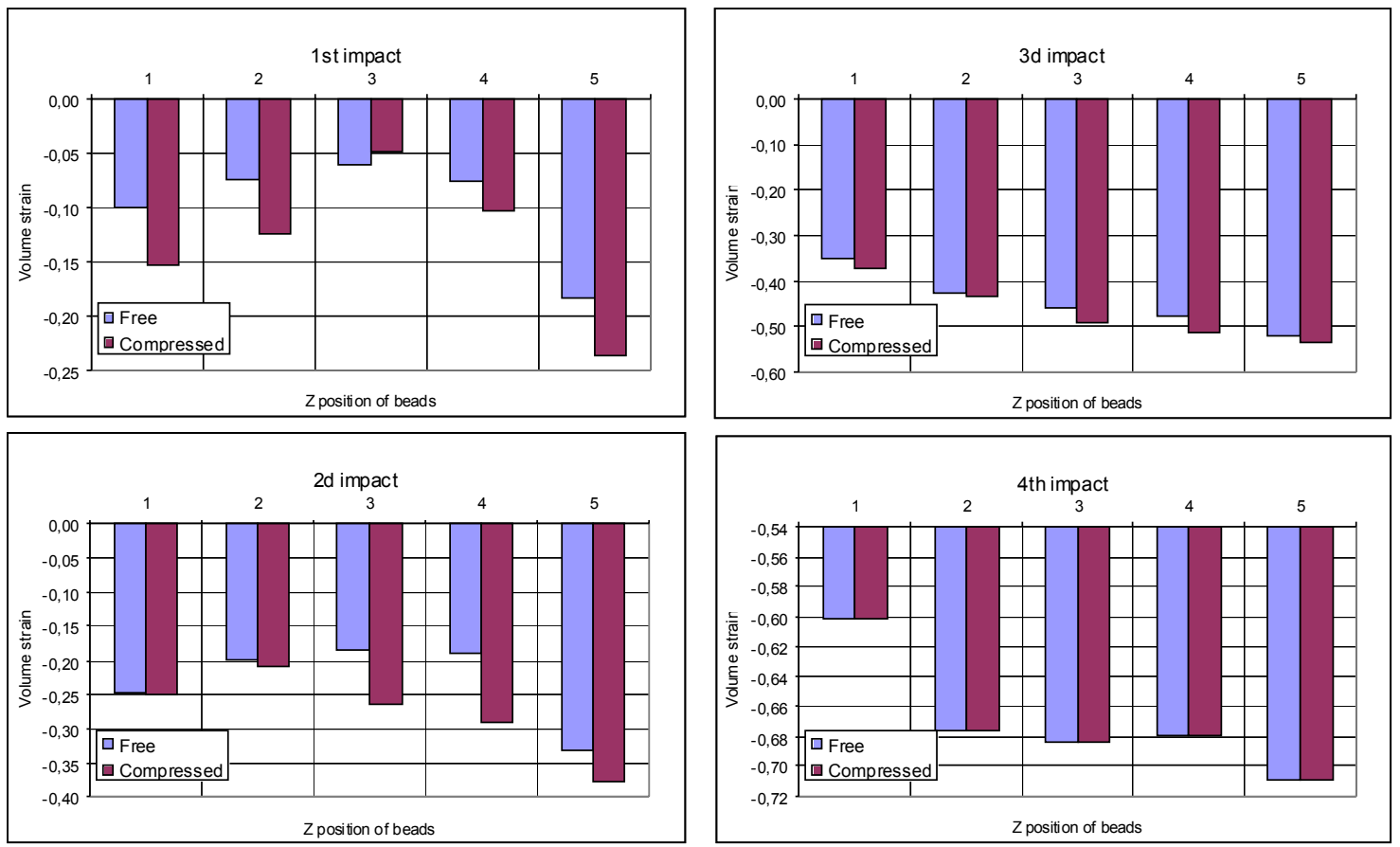

Figure 17: Volume strain as a function of bead vertical $(Z)$ position 


\begin{tabular}{|c|c|c|r|r|r|r|r|r|}
\hline & \multicolumn{2}{|c|}{ 1st impact } & \multicolumn{2}{c|}{ 2d impact } & \multicolumn{2}{c|}{ 3d impact } & \multicolumn{2}{c|}{ 4th impact } \\
\hline & compressed & unloaded & compressed & unloaded & compressed & unloaded & compressed & unloaded \\
\hline $\begin{array}{c}\text { Internal } \\
\text { zone }\end{array}$ & -0.24 & -0.17 & $-0,43$ & $-0,38$ & $-0,64$ & $-0,63$ & $-0,82$ & $-0,76$ \\
\hline $\begin{array}{c}\text { External } \\
\text { zone }\end{array}$ & -0.07 & -0.06 & $-0,18$ & $-0,14$ & $-0,35$ & $-0,32$ & $-0,57$ & $-0,47$ \\
\hline
\end{tabular}

Table 1: Volume strain mean as a function of the radial position (internal or external) of beads for each impact

\begin{tabular}{|c|c|c|c|c|c|c|c|c|}
\hline \multirow{3}{*}{$\begin{array}{l}\text { Bead } \\
\text { number }\end{array}$} & & & \multicolumn{2}{|c|}{ 2nd impact } & \multicolumn{2}{|c|}{ 3rd impact } & \multicolumn{2}{|c|}{ 4th impact } \\
\hline & & & unloaded & compressed & unloaded & compressed & unloaded & compressed \\
\hline & & Density & Vol. strain & Vol. strain & Vol. strain & Vol. strain & Vol. strain & Vol. strain \\
\hline & 45 & 84,2 & $-0,22$ & $-0,26$ & $-0,49$ & $-0,56$ & $-0,61$ & $-0,69$ \\
\hline & 61 & 93,8 & $-0,13$ & $-0,17$ & $-0,43$ & $-0,44$ & $-0,56$ & $-0,64$ \\
\hline & 31 & 178,7 & $-0,16$ & $-0,18$ & $-0,29$ & $-0,37$ & $-0,48$ & $-0,53$ \\
\hline & 41 & 178,8 & $-0,06$ & $-0,15$ & $-0,28$ & $-0,29$ & $-0,40$ & $-0,53$ \\
\hline
\end{tabular}

Table 2: Volume strain for four beads calculated for the second, third and fourth impact (in the compressed and unloaded states) 